\title{
Geometric Mappings under the Perturbed Extension Operators in Complex Systems Analysis
}

\author{
Chaojun Wang, ${ }^{1}$ Yanyan Cui, ${ }^{1,2}$ and Hao Liu $^{3}$ \\ ${ }^{1}$ College of Mathematics and Statistics, Zhoukou Normal University, Zhoukou, Henan 466001, China \\ ${ }^{2}$ College of Mathematics and Information Science, Hebei Normal University, Shijiazhuang, Hebei 050016, China \\ ${ }^{3}$ Institute of Contemporary Mathematics, Henan University, Kaifeng, Henan 475001, China \\ Correspondence should be addressed to Yanyan Cui; cui9907081@163.com
}

Received 3 March 2017; Accepted 8 May 2017; Published 15 June 2017

Academic Editor: Chris Goodrich

Copyright (C) 2017 Chaojun Wang et al. This is an open access article distributed under the Creative Commons Attribution License, which permits unrestricted use, distribution, and reproduction in any medium, provided the original work is properly cited.

\begin{abstract}
In this paper, we mainly seek conditions on which the geometric properties of subclasses of biholomorphic mappings remain unchanged under the perturbed Roper-Suffridge extension operators. Firstly we generalize the Roper-Suffridge operator on Bergman-Hartogs domains. Secondly, applying the analytical characteristics and growth results of subclasses of biholomorphic mappings, we conclude that the generalized Roper-Suffridge operators preserve the geometric properties of strong and almost spiral-like mappings of type $\beta$ and order $\alpha, S_{\Omega}^{*}(\beta, A, B)$ as well as almost spiral-like mappings of type $\beta$ and order $\alpha$ under different conditions on Bergman-Hartogs domains. Sequentially we obtain the conclusions on the unit ball $B^{n}$ and for some special cases. The conclusions include and promote some known results and provide new approaches to construct biholomorphic mappings which have special geometric characteristics in several complex variables.
\end{abstract}

\section{Introduction}

The theory of several complex variables derives from the theory of one complex variable. There are many excellent results in geometric function theories of one complex variable. It is natural to think that we can extend these results in several complex variables, while some basic theorems (such as the models of the coefficients of the homogeneous expansion for biholomorphic functions being bounded on the unit disk [1]) are found not to hold in several complex variables. In 1933, Cartan [2] suggested that we can consider the geometric constraint of biholomorphic mappings, such as star-likeness and convexity. So many scholars devoted themselves to the research of star-like mappings and convex mappings. Recently many subclasses or expansions of starlike and convex mappings are introduced. The properties of biholomorphic mappings with special geometric properties are important research objects in geometric function theories of several complex variables. It is easy to find specific examples of these new subclasses or expansions in $\mathbb{C}$, while it is very difficult in $\mathbb{C}^{n}$. In order to study these subclasses better in several complex variables, we need the specific examples imminently.

In 1995, Roper and Suffridge [3] introduced an operator

$$
\phi_{n}(f)(z)=\left(f\left(z_{1}\right), \sqrt{f^{\prime}\left(z_{1}\right)} z_{0}\right)^{\prime},
$$

where $z=\left(z_{1}, z_{0}\right) \in B^{n}, z_{1} \in D, z_{0}=\left(z_{2}, \ldots, z_{n}\right) \in$ $\mathbb{C}^{n-1}, f\left(z_{1}\right) \in H(D), \sqrt{f^{\prime}(0)}=1$. Roper and Suffridge proved the Roper-Suffridge operator preserves convexity and star-likeness on $B^{n}$. Graham et al. generalized the RoperSuffridge operator and discussed the generalized operators preserving star-likeness and the block property in $[4,5]$. In 2002, Graham et al. extended the Roper-Suffridge operator on the unit ball in $\mathbb{C}^{n}$ and proved the extended operator preserves star-likeness and convexity if and only if some conditions are satisfied in [6]. In 2003, Gong and Liu [7] generalized the Roper-Suffridge operator and obtained the generalized operator preserving $\varepsilon$ star-likeness on the Reinhardt domain which leads to star-likeness and convexity in the cases of $\varepsilon=0$ and $\varepsilon=1$, respectively. 
All of the above illustrate that the Roper-Suffridge operator has good properties. Through the Roper-Suffridge operator or its generalizations we can construct lots of convex mappings and star-like mappings in $\mathbb{C}^{n}$ by corresponding functions on the unit disk $D$ of $\mathbb{C}$. That will promote the development of the research of biholomorphic mappings. So the Roper-Suffridge operator plays an important role in several complex variables. In recent years, there are lots of results about the Roper-Suffridge extension operator which was generalized and modified on different domains in different spaces to preserve the geometric characteristics of convex mappings, star-like mappings, and their subclasses. Graham and Kohr gave a survey about the Roper-Suffridge extension operator and the developments in the theory of biholomorphic mappings in several complex variables to which it had led in [8].

Muir and Suffridge [9] introduced the following generalized Roper-Suffridge extension operator:

$$
F(z)=\left(f\left(z_{1}\right)+f^{\prime}\left(z_{1}\right) P\left(z_{0}\right), \sqrt{f^{\prime}\left(z_{1}\right)} z_{0}\right)^{\prime},
$$

where $f$ is a normalized biholomorphic function on the unit disk $D, z=\left(z_{1}, z_{0}\right)^{\prime} \in B^{n}, z_{1} \in D, z_{0}=\left(z_{2}, \ldots, z_{n}\right)^{\prime} \in$ $\mathbb{C}^{n-1}$. The branch of the power function is chosen such that $\sqrt{f^{\prime}(0)}=1 . P: \mathbb{C}^{n-1} \rightarrow \mathbb{C}$ is a homogeneous polynomial of degree 2 . The extended operator (2) was proved to preserve star-likeness on $\|P\| \leq 1 / 4$ and convexity on $\|P\| \leq 1 / 2$ in [9] and was proved to take the extreme points of normalized convex functions to extreme points of normalized convex mappings of the Euclidean ball in $\mathbb{C}^{n}$ under precise conditions by Muir in [10]. Also the extended operator (2) was studied by Kohr and Muir in [11, 12] with Loewner chains. Later the operator (2) was generalized by Elin and Levenshtein and the generalized operator was proved to preserve the spirallikeness property in [13] and was concluded that it can be embedded in a Loewner chain on the unit ball in $\mathbb{C}^{n}$ in [14]. Moreover, Elin and Levenshtein presented an extension operator for semigroup generators and concluded that the new one-dimensional covering results established in [13] are crucial. Furthermore, (2) was modified and discussed by Cui et al. in [15]. Elin introduced a general construction of the extension operators

$$
\phi_{\Gamma}[h](x, y)=(h(x), \Gamma((h, x) y)),
$$

where $(x, y)$ is on the unit ball of the product $X * Y$ of two Banach spaces and $\Gamma(h, x)$ is an operator-valued mapping which satisfies some natural conditions. $\phi_{\Gamma}[h](x, y)$ was proved to preserve star-likeness and spiral-likeness under some conditions in [16].

Now, we introduce a new extension operator

$$
\begin{aligned}
& F(w, z) \\
& \quad=\left(w_{(1)}\left(\frac{f\left(z_{1}\right)}{z_{1}}\right)^{\delta_{1}}, \ldots, w_{(s)}\left(\frac{f\left(z_{1}\right)}{z_{1}}\right)^{\delta_{s}}, f\left(z_{1}\right)\right. \\
& \left.\quad+G\left[\left(\frac{f\left(z_{1}\right)}{z_{1}}\right)^{\gamma} z_{0}\right],\left(\frac{f\left(z_{1}\right)}{z_{1}}\right)^{\gamma} z_{0}\right)^{\prime}
\end{aligned}
$$

on the Bergman-Hartogs domain

$$
\begin{aligned}
& \Omega_{p_{1}, \ldots, p_{s}, q}^{B^{n}}=\left\{\left(w_{(1)}, \ldots, w_{(s)}, z\right) \in \mathbb{C}^{m_{1}} \times \cdots \times \mathbb{C}^{m_{s}}\right. \\
& \left.\quad \times B^{n}:\left\|w_{(1)}\right\|^{2 p_{1}}+\cdots+\left\|w_{(s)}\right\|^{2 p_{s}}<K_{B^{n}}(z, z)^{-q}\right\},
\end{aligned}
$$

where $(w, z) \in \Omega_{p_{1}, \ldots, p_{s}, q}^{B^{n}}, z=\left(z_{1}, z_{0}\right) \in B^{n}, f\left(z_{1}\right)$ is a normalized univalent holomorphic function on $D, G$ is a holomorphic function in $\mathbb{C}^{n-1}$ with $G(0)=0, D G(0)=$ I, $\gamma \geq 0$, and the power functions take the branches such that $\left.\left[f\left(z_{1}\right) / z_{1}\right]^{\delta_{i}}\right|_{z_{1}=0}=1(i=1, \ldots, s)$ and $\left.\left[f\left(z_{1}\right) / z_{1}\right]^{\gamma}\right|_{z_{1}=0}=1$. The homogeneous expansion of $G(z)$ is $\sum_{j=0}^{\infty} P_{j}(z)$, where $P_{j}(z)$ is a homogeneous polynomial of degree $j$. Equation (4) is the modification of the extension operators discussed in [17].

In the case of $w_{(1)}=\cdots=w_{(s)}=0$, (4) leads to the following operator:

$$
\begin{aligned}
& F(z) \\
& \quad=\left(f\left(z_{1}\right)+G\left[\left(\frac{f\left(z_{1}\right)}{z_{1}}\right)^{\gamma} z_{0}\right],\left(\frac{f\left(z_{1}\right)}{z_{1}}\right)^{\gamma} z_{0}\right)^{\prime},
\end{aligned}
$$

which can also be seen as the modification of the following generalized Roper-Suffridge extension operator introduced by Muir on the unit ball in complex Banach spaces,

$$
\begin{aligned}
& {\left[\Phi_{G, \gamma}(f)\right](z)} \\
& \quad=\left(f\left(z_{1}\right)+G\left(\left[f^{\prime}\left(z_{1}\right)\right]^{\gamma} z_{0}\right),\left[f^{\prime}\left(z_{1}\right)\right]^{\gamma} z_{0}\right)^{\prime},
\end{aligned}
$$

where $\left.\left[f^{\prime}\left(z_{1}\right)\right]^{\gamma}\right|_{z_{1}=0}=1$. Muir proved $\left[\Phi_{G, \gamma}(f)\right](z)$ is a Loewner chain preserving extension operator provided that $G$ satisfies some conditions in [12].

In this paper, we mainly discuss the invariance of several biholomorphic mappings under the generalized RoperSuffridge extension operators (4) on the Bergman-Hartogs domains $\Omega_{p_{1}, \ldots, p_{s}, q}^{B^{n}}$ which is based on the unit ball $B^{n}$. In Section 2, we give some definitions and lemmas that are used to derive the main results. In Sections 3-5, we detailedly discuss the perturbed Extension Operator (4) preserving the geometric properties of strong and almost spiral-like mappings of type $\beta$ and order $\alpha, S_{\Omega}^{*}(\beta, A, B)$, as well as almost spiral-like mappings of type $\beta$ and order $\alpha$ under different conditions on Bergman-Hartogs domains and thus generalize the conclusions on the unit ball $B^{n}$ in $\mathbb{C}^{n}$. At last, we derive that the generalized Roper-Suffridge operators preserve the properties of subclasses of the three kinds of biholomorphic mappings mentioned above. The conclusions include and promote some known results.

\section{Definitions and Lemmas}

In the following, let $D$ denote the unit disk in $\mathbb{C}$ and $B^{n}$ denote the unit ball in $\mathbb{C}^{n}$. Let $D F(z)$ denote the Fréchet derivative of $F$ at $z$.

To get the main results, we need the following definitions and lemmas. 
Definition 1 (see [18]). Let $\Omega$ be a bounded star-like circular domain in $\mathbb{C}^{n}$. The Minkowski functional $\rho(z)$ of $\Omega$ is $C^{1}$ except for a lower-dimensional manifold. If $f(z)$ is a normalized locally biholomorphic mapping on $\Omega$, let $\alpha \epsilon$ $[0,1), \beta \in(-\pi / 2, \pi / 2)$, and

$$
\begin{aligned}
& \mid \frac{-\alpha+i \tan \beta}{1-\alpha} \\
& \quad+\frac{1-i \tan \beta}{1-\alpha} \frac{2}{\rho(z)} \frac{\partial \rho}{\partial z}(z)(D f(z))^{-1} f(z)-\frac{1+c^{2}}{1-c^{2}} \mid \\
& \quad<\frac{2 c}{1-c^{2}}
\end{aligned}
$$

Then $f(z)$ is called a strong and almost spiral-like mapping of type $\beta$ and order $\alpha$ on $\Omega$.

Setting $\alpha=0, \beta=0$, and $\alpha=\beta=0$, Definition 1 reduces to the definition of strong spiral-like mappings of type $\beta$, strong and almost star-like mappings of order $\alpha$, and strong star-like mappings, respectively.

Definition 2 (see [19]). Let $\Omega$ be a bounded star-like circular domain in $\mathbb{C}^{n}$. The Minkowski functional $\rho(z)$ of $\Omega$ is $C^{1}$ except for a lower-dimensional manifold. Let $F(z)$ be a normalized locally biholomorphic mapping on $\Omega$. If

$$
\begin{gathered}
\mid i \tan \beta+(1-i \tan \beta) \frac{2}{\rho(z)} \frac{\partial \rho}{\partial z}(z) J_{F}^{-1}(z) F(z) \\
-\frac{1-A B}{1-B^{2}} \mid<\frac{B-A}{1-B^{2}}, \quad z \in \Omega \backslash\{0\},
\end{gathered}
$$

where $-1 \leq A<B<1$ and $\beta \in(-\pi / 2, \pi / 2)$, then we call $F \in S_{\Omega}^{*}(\beta, A, B)$.

Setting $A=-1=-B-2 \alpha, A=-B=-\alpha$, and $B \rightarrow 1^{-}$ in Definition 2, respectively, we obtain the corresponding definitions of spiral-like mappings of type $\beta$ and order $\alpha$, strong spiral-like mappings of type $\beta$ and order $\alpha$, and almost spiral-like mappings of type $\beta$ and order $\alpha$ on $\Omega$.

Definition 3 (see [20]). Let $\Omega$ be a bounded star-like circular domain in $\mathbb{C}^{n}$. The Minkowski functional $\rho(z)$ of $\Omega$ is $C^{1}$ except for a lower-dimensional manifold. Let $f(z)$ be a normalized locally biholomorphic mapping on $\Omega$. If

$$
\begin{array}{r}
\mathfrak{R}\left[e^{-i \beta} \frac{2}{\rho(z)} \frac{\partial \rho}{\partial z}(z)(D f(z))^{-1} f(z)\right] \geq \\
\\
z \in \Omega \cos \beta, \\
z 0\},
\end{array}
$$

where $\alpha \in[0,1)$ and $\beta \in(-\pi / 2, \pi / 2)$, then we call $f(z)$ an almost spiral-like mapping of type $\beta$ and order $\alpha$.

Setting $\alpha=0, \beta=0$ in Definition 3, we obtain the definition of spiral-like mappings of type $\beta$ and almost starlike mappings of order $\alpha$ on $B^{n}$, respectively.
Lemma 4 (see [21]). Let $\Omega$ be a bounded star-like circular domain in $\mathbb{C}^{n}$. The Minkowski functional $\rho(z)$ of $\Omega$ is $C^{1}$ except for a lower-dimensional manifold $\Omega_{0}$. Then we have

$$
\begin{aligned}
2 \frac{\partial \rho(z)}{\partial z} z & =\rho(z), \\
\frac{\partial \rho}{\partial z}(\lambda z) & =\frac{\partial \rho(z)}{\partial z} \quad(\lambda \geq 0), \\
\frac{\partial \rho}{\partial z}\left(e^{i \theta} z\right) & =e^{-i \theta} \frac{\partial \rho(z)}{\partial z} \quad(\theta \in R) .
\end{aligned}
$$

Lemma 5 (see [12]). Let $P(z)$ be a homogeneous polynomial of degree $m$ and let $D P(z)$ be the Fréchet derivative of $P$ at $z$. Then

$$
D P(z) z=m P(z)
$$

Lemma 6 (see [17]). Let $\rho(w, z)$ be the Minkowski functional of $\Omega_{p_{1}, \ldots, p_{s}, q}^{B^{n}}$. Let $(w, z) \in \partial \Omega_{p_{1}, \ldots, p_{s}, q}^{B^{n}}$. Then $\rho(w, z)=1$ and

$$
\begin{aligned}
& \frac{\partial \rho(w, z)}{\partial w_{i j}}=\frac{p_{i}\left\|w_{(i)}\right\|^{2 p_{i}-2} \overline{w_{i j}}}{2 \nabla_{1}+2 \nabla_{2}}, \\
& \frac{\partial \rho(w, z)}{\partial z_{i}}=\frac{\nabla_{1}\left(\overline{z_{i}} /\|z\|^{2}\right)}{2 \nabla_{1}+2 \nabla_{2}},
\end{aligned}
$$

$$
i=1, \ldots, s, j=1, \ldots, m_{i}
$$

where

$$
\begin{aligned}
& \nabla_{1}=(n+1) q \pi^{n q}(n !)^{-q}\left(1-\|z\|^{2}\right)^{(n+1) q-1}\|z\|^{2}, \\
& \nabla_{2}=\sum_{k=1}^{s} p_{k}\left\|w_{(k)}\right\|^{2 p_{k}} .
\end{aligned}
$$

Lemma 7 (see [1]). If $f(z)$ is a normalized biholomorphic function on the unit disk $D$, then

$$
\frac{|z|}{(1+|z|)^{2}} \leq|f(z)| \leq \frac{|z|}{(1-|z|)^{2}} .
$$

Lemma 8 (see [22]). Let $f(z)$ be a strong spiral-like mapping of type $\alpha$ on bounded and balanced domain $\Omega$ with $\alpha \epsilon$ $(-\pi / 2, \pi / 2)$ and $c \in(0,1)$. Let the Minkowski functional of $\Omega$ be $\rho(z)$. Then

$$
\frac{\rho(z)}{(1+c \rho(z))^{2}} \leq \rho(f(z)) \leq \frac{\rho(z)}{(1-c \rho(z))^{2}} .
$$

Let $f(z)$ be a strong spiral-like function of type $\alpha$ on $D$, then

$$
\frac{|z|}{(1+c|z|)^{2}} \leq|f(z)| \leq \frac{|z|}{(1-c|z|)^{2}} .
$$

Lemma 9 (see [23]). Let $\Omega \subset \mathbb{C}^{n}$ be a bounded star-like circular domain and the Minkowski functional $\rho(z)$ of $\Omega$ be $C^{1}$ 
except for some submanifolds of lower dimensions. Let $f(z) \in$ $S_{\Omega}^{*}(A, B)$ be $k$-fold symmetric. Then

$$
\begin{aligned}
\frac{\rho(z)}{(1-A \rho(z))^{(A-B) / A}} & \leq \rho(f(z)) \\
& \leq \frac{\rho(z)}{(1+A \rho(z))^{(A-B) / A}}, \quad A \neq 0 \\
\rho(z) e^{-B \rho(z)} & \leq \rho(f(z)) \leq \rho(z) e^{B \rho(z)}, \\
& \\
& A=0,
\end{aligned}
$$

or, equivalently,

$$
\begin{gathered}
\frac{|z|}{(1-A|z|)^{(A-B) / A}} \leq|f(z)| \leq \frac{|z|}{(1+A|z|)^{(A-B) / A}}, \\
\quad A \neq 0 \\
|z| e^{-B|z|} \leq|f(z)| \leq|z| e^{B|z|}, \quad A=0 .
\end{gathered}
$$

The above estimates are all accurate.

Lemma 10 (see [24]). Let $f(x)$ be an almost spiral-like mapping of type $\beta$ and order $\alpha$ on the unit ball $B$ in complex Banach spaces with $\alpha \in[0,1)$ and $\beta \in(-\pi / 2, \pi / 2)$. Then

$$
\begin{gathered}
\frac{\|x\|}{[1+(1-2 \alpha)\|x\|]^{2(\alpha-1) /(2 \alpha-1)}} \leq\|f(x)\| \\
\leq \frac{\|x\|}{[1-(1-2 \alpha)\|x\|]^{2(\alpha-1) /(2 \alpha-1)}} .
\end{gathered}
$$

For $n=1$ we get

$$
\begin{gathered}
\frac{|z|}{[1+(1-2 \alpha)|z|]^{2(\alpha-1) /(2 \alpha-1)}}|f(z)| \\
\leq \frac{|z|}{[1-(1-2 \alpha)|z|]^{2(\alpha-1) /(2 \alpha-1)}}
\end{gathered}
$$

\section{The Invariance of Strong and Almost Spiral- Like Mappings of Type $\beta$ and Order $\alpha$}

For simplicity, let $\Omega$ denote $\Omega_{p_{1}, \ldots, p_{s}, q}^{B^{n}}$. In this section we will show that the perturbed Roper-Suffridge extension operator (4) preserves the geometric characteristics of strong and almost spiral-like mappings of type $\beta$ and order $\alpha$ on $\Omega$, and thus we obtain the conclusion on $B^{n}$; also we get the invariance of some subclasses.

Theorem 11. Let $f\left(z_{1}\right)$ be a strong and almost spiral-like function of type $\beta$ and order $\alpha$ on $D$ with $\alpha \in[0,1)$ and $\beta \in(-\pi / 2, \pi / 2), c \in(0,1)$. Let $F(w, z)$ be the mapping denoted by (4) with $p_{i}>1, \delta_{i} \in[0,1](i=1, \ldots, s), \gamma<1 / 4$ and $\left.\left(f\left(z_{1}\right) / z_{1}\right)^{\delta_{i}}\right|_{z_{1}=0}=1,\left.\left(f\left(z_{1}\right) / z_{1}\right)^{\gamma}\right|_{z_{1}=0}=1$. Then $F(w, z)$ is a strong and almost spiral-like mapping of type $\beta$ and order $\alpha$ on $\Omega$ provided that $P_{j}=0$ for $j<2 /(1-4 \gamma)$ and

$$
\begin{aligned}
q & \geq \frac{\delta}{n+1}, \\
\sum_{j=2}^{\infty} 2^{j / 2+1}(j-1)\left\|P_{j}\right\| & \leq \frac{2 c(1-\gamma)(1-\alpha) \cos \beta}{(1+c)(1+(1-\alpha) \cos \beta)},
\end{aligned}
$$

where $\delta=\max \left\{p_{1} \delta_{1}, \ldots, p_{s} \delta_{s}\right\}$.

Proof. By Definition 1, we need to prove

$$
\begin{gathered}
\mid \frac{1-c^{2}}{2 c} \frac{1-i \tan \beta}{1-\alpha} \frac{2}{\rho(w, z)} \frac{\partial \rho(w, z)}{\partial(w, z)}(D F(w, z))^{-1} \\
\cdot F(w, z)+\frac{1-c^{2}}{2 c} \frac{-\alpha+i \tan \beta}{1-\alpha}-\frac{1+c^{2}}{2 c} \mid<1 .
\end{gathered}
$$

It is obvious that (23) holds for $w=z_{0}=0$. Otherwise, setting $(w, z)=\zeta(\xi, \eta)=|\zeta| e^{i \theta}(\xi, \eta)$ where $(\xi, \eta) \in \partial \Omega$ and $\zeta \in \bar{D} \mid$ $\{0\}$, by Lemma 4 , we obtain

$$
\begin{aligned}
& \frac{2}{\rho(w, z)} \frac{\partial \rho(w, z)}{\partial(w, z)}(D F(w, z))^{-1} F(w, z) \\
& =\frac{2}{\rho\left(|\zeta| e^{i \theta}(\xi, \eta)\right)} \frac{\partial \rho}{\partial(w, z)}\left(|\zeta| e^{i \theta}(\xi, \eta)\right) \\
& \cdot(D F(\zeta \xi, \zeta \eta))^{-1} F(\zeta \xi, \zeta \eta)=\frac{2}{|\zeta|} \frac{e^{-i \theta} \partial \rho}{\partial(w, z)}(\xi, \eta) \\
& \cdot(D F(\zeta \xi, \zeta \eta))^{-1} F(\zeta \xi, \zeta \eta)=\frac{2 \partial \rho}{\partial(w, z)}(\xi, \eta) \\
& \cdot \frac{(D F(\zeta \xi, \zeta \eta))^{-1} F(\zeta \xi, \zeta \eta)}{\zeta} .
\end{aligned}
$$

Fix $\xi, \eta$, then $(2 \partial \rho / \partial(w, z))(\xi, \eta)\left((D F(\zeta \xi, \zeta \eta))^{-1} F(\zeta \xi, \zeta \eta) / \zeta\right)$ is holomorphic with respect to $\zeta$. Due to the maximum modulus principle of holomorphic functions, the left side of (23) gets the maximum value at $|\zeta|=1$. So we need only to prove that $(23)$ holds for $(w, z) \in \partial \Omega$, which follows $\rho(w, z)=$ 1.

Let

$$
\begin{aligned}
q\left(z_{1}\right)= & \frac{1-c^{2}}{2 c} \frac{1-i \tan \beta}{1-\alpha} \frac{f\left(z_{1}\right)}{z_{1} f^{\prime}\left(z_{1}\right)} \\
& +\frac{1-c^{2}}{2 c} \frac{-\alpha+i \tan \beta}{1-\alpha}-\frac{1+c^{2}}{2 c} .
\end{aligned}
$$

Then $q\left(z_{1}\right) \in H(D),\left|q\left(z_{1}\right)\right|<1$ and $q(0)=-c$. Letting $g\left(z_{1}\right)=\left(2 c /\left(1-c^{2}\right)\right)\left(q\left(z_{1}\right)+c\right)$, we have $g\left(z_{1}\right) \in H(D), g(0)=$ 0 and $\left|q\left(z_{1}\right)\right|=\left|\left(\left(1-c^{2}\right) / 2 c\right) g\left(z_{1}\right)-c\right|<1$. In view of $\left(\left(1-c^{2}\right) / 2 c\right)\left|g\left(z_{1}\right)\right|-c<\left|\left(\left(1-c^{2}\right) / 2 c\right) g\left(z_{1}\right)-c\right|$, we obtain $\left|g\left(z_{1}\right)\right|<2 c /(1-c)<1$ provided that $c \leq 1 / 3$. By Schwarz Lemma we get $\left|g\left(z_{1}\right)\right| \leq\left|z_{1}\right|$ which implies that

$$
\left|q\left(z_{1}\right)+c\right| \leq \frac{1-c^{2}}{2 c}\left|z_{1}\right| .
$$

In addition, from (4) we have 


$$
D F(w, z)=\left\{\begin{array}{cccccc}
\left(\frac{f\left(z_{1}\right)}{z_{1}}\right)^{\delta_{1}} & 0 & \ldots & 0 & v_{1} & 0_{1 \times(n-1)} \\
\cdots & \ldots & \ldots & \ldots & \cdots & \cdots \\
0 & 0 & \cdots & \left(\frac{f\left(z_{1}\right)}{z_{1}}\right)^{\delta_{s}} & v_{s} & 0_{1 \times(n-1)} \\
0 & 0 & \cdots & 0 & u_{1} & \sum_{j=2}^{\infty}\left(\frac{f\left(z_{1}\right)}{z_{1}}\right)^{\gamma_{j}} D P_{j}\left(z_{0}\right) \\
0_{(n-1) \times 1} & 0_{(n-1) \times 1} & \cdots & 0_{(n-1) \times 1} & \gamma\left(\frac{f\left(z_{1}\right)}{z_{1}}\right)^{\gamma} \frac{z_{1} f^{\prime}-f}{z_{1} f} z_{0}^{\prime} & \left(\frac{f\left(z_{1}\right)}{z_{1}}\right)^{\gamma} I_{n-1}
\end{array}\right\},
$$

where

$$
\begin{aligned}
& v_{i}=\delta_{i} w_{(i)}\left(\frac{f\left(z_{1}\right)}{z_{1}}\right)^{\delta_{i}} \frac{z_{1} f^{\prime}-f}{z_{1} f} \quad(i=1, \ldots, s), \\
& u_{1}=f^{\prime}+\frac{z_{1} f^{\prime}-f}{z_{1} f} \sum_{j=2}^{\infty} \gamma j\left(\frac{f\left(z_{1}\right)}{z_{1}}\right)^{\gamma j} P_{j}\left(z_{0}\right) .
\end{aligned}
$$

Let $(D F(w, z))^{-1} F(w, z)=H(w, z)=\left(h_{1}, \ldots, h_{s+n}\right)^{\prime}$, which follows $D F(w, z)\left(h_{1}, \ldots, h_{s+n}\right)^{\prime}=F(w, z)$. Thus

$$
\begin{gathered}
\left(\frac{f}{z_{1}}\right)^{\delta_{i}} h_{i}+v_{i} h_{s+1}=w_{(i)}\left(\frac{f\left(z_{1}\right)}{z_{1}}\right)^{\delta_{i}}, \quad i=1, \ldots, s \\
u_{1} h_{s+1}+\sum_{j=2}^{\infty}\left(\frac{f\left(z_{1}\right)}{z_{1}}\right)^{\gamma j} D P_{j}\left(z_{0}\right)\left(h_{s+2}, \ldots, h_{s+n}\right)^{\prime} \\
=f\left(z_{1}\right)+\sum_{j=2}^{\infty}\left(\frac{f\left(z_{1}\right)}{z_{1}}\right)^{\gamma j} P_{j}\left(z_{0}\right) \\
\gamma\left(\frac{f\left(z_{1}\right)}{z_{1}}\right)^{\gamma} \frac{z_{1} f^{\prime}-f}{z_{1} f} z_{j} h_{s+1}+\left(\frac{f}{z_{1}}\right)^{\gamma} h_{s+j} \\
=\left(\frac{f\left(z_{1}\right)}{z_{1}}\right)^{\gamma} z_{j}, \quad j=2, \ldots, n .
\end{gathered}
$$

Lemma 5 and a straightforward calculation show that

$$
\begin{aligned}
h_{i} & =w_{(i)}\left[1-\delta_{i} \frac{z_{1} f^{\prime}-f}{z_{1} f^{\prime}}(1\right. \\
& \left.\left.+\frac{1}{f} \sum_{j=2}^{\infty}\left(\frac{f\left(z_{1}\right)}{z_{1}}\right)^{\gamma j}(1-j) P_{j}\left(z_{0}\right)\right)\right],
\end{aligned}
$$

$$
\begin{aligned}
h_{s+1} & =\frac{f}{f^{\prime}}+\frac{1}{f^{\prime}} \sum_{j=2}^{\infty}\left(\frac{f\left(z_{1}\right)}{z_{1}}\right)^{\gamma j}(1-j) P_{j}\left(z_{0}\right) \\
h_{s+j} & =z_{j}\left[1-\gamma \frac{z_{1} f^{\prime}-f}{z_{1} f^{\prime}}(1\right. \\
& \left.\left.+\frac{1}{f} \sum_{j=2}^{\infty}\left(\frac{f\left(z_{1}\right)}{z_{1}}\right)^{\gamma j}(1-j) P_{j}\left(z_{0}\right)\right)\right],
\end{aligned}
$$$$
j=2, \ldots, n .
$$

As a consequence of Lemma 6 we get

$$
\frac{2 \partial \rho}{\partial(w, z)}(w, z)(D F(w, z))^{-1} F(w, z)=\frac{G(w, z)}{\nabla_{1}+\nabla_{2}},
$$

where

$$
\begin{aligned}
& G(w, z)=\sum_{k=1}^{s} p_{k}\left\|w_{(k)}\right\|^{2 p_{k}}\left[1-\delta_{k}\right. \\
& \left.. \frac{z_{1} f^{\prime}-f}{z_{1} f^{\prime}}\left(1+\frac{1}{f} \sum_{j=2}^{\infty}\left(\frac{f}{z_{1}}\right)^{\gamma j}(1-j) P_{j}\left(z_{0}\right)\right)\right] \\
& +\frac{\left|z_{1}\right|^{2} \nabla_{1}}{\|z\|^{2}} \frac{f}{z_{1} f^{\prime}}\left[1+\frac{1}{f} \sum_{j=2}^{\infty}\left(\frac{f}{z_{1}}\right)^{\gamma j}(1-j) P_{j}\left(z_{0}\right)\right] \\
& +\frac{\left\|z_{0}\right\|^{2} \nabla_{1}}{\|z\|^{2}}[1-\gamma \\
& \left.. \frac{z_{1} f^{\prime}-f}{z_{1} f^{\prime}}\left(1+\frac{1}{f} \sum_{j=2}^{\infty}\left(\frac{f}{z_{1}}\right)^{\gamma j}(1-j) P_{j}\left(z_{0}\right)\right)\right] .
\end{aligned}
$$

Let

$$
\begin{gathered}
\frac{1-c^{2}}{2 c} \frac{1-i \tan \beta}{1-\alpha} \frac{2 \partial \rho(w, z)}{\partial(w, z)}(D F(w, z))^{-1} F(w, z) \\
+\frac{1-c^{2}}{2 c} \frac{-\alpha+i \tan \beta}{1-\alpha}-\frac{1+c^{2}}{2 c}=I .
\end{gathered}
$$


Taking into account (25) and (31) we obtain the following equation string:

$$
\begin{aligned}
& \left(\nabla_{1}+\nabla_{2}\right) I=\frac{1-c^{2}}{2 c} \frac{1-i \tan \beta}{1-\alpha}\left\{\sum_{k=1}^{s} p_{k}\left\|w_{(k)}\right\|^{2 p_{k}}[1\right. \\
& -\delta_{k} \\
& \left.\cdot \frac{z_{1} f^{\prime}-f}{z_{1} f^{\prime}}\left(1+\frac{1}{f} \sum_{j=2}^{\infty}\left(\frac{f}{z_{1}}\right)^{\gamma j}(1-j) P_{j}\left(z_{0}\right)\right)\right] \\
& +\frac{\left|z_{1}\right|^{2} \nabla_{1}}{\|z\|^{2}} \frac{f}{z_{1} f^{\prime}}\left[1+\frac{1}{f} \sum_{j=2}^{\infty}\left(\frac{f}{z_{1}}\right)^{\gamma j}(1-j) P_{j}\left(z_{0}\right)\right] \\
& +\frac{\left\|z_{0}\right\|^{2} \nabla_{1}}{\|z\|^{2}}\left[1-\gamma \frac{z_{1} f^{\prime}-f}{z_{1} f^{\prime}}\right. \\
& \left.\left.\cdot\left(1+\frac{1}{f} \sum_{j=2}^{\infty}\left(\frac{f}{z_{1}}\right)^{\gamma j}(1-j) P_{j}\left(z_{0}\right)\right)\right]\right\} \\
& +\left(\frac{1-c^{2}}{2 c} \frac{-\alpha+i \tan \beta}{1-\alpha}-\frac{1+c^{2}}{2 c}\right)\left(\nabla_{1}+\nabla_{2}\right) \\
& =\frac{1-c^{2}}{2 c} \frac{1-i \tan \beta}{1-\alpha} \nabla_{2}+\widetilde{\nabla_{2}}\left(q\left(z_{1}\right)+c\right)\left[1+\frac{1}{f}\right. \\
& \left.\cdot \sum_{j=2}^{\infty}\left(\frac{f}{z_{1}}\right)^{\gamma j}(1-j) P_{j}\left(z_{0}\right)\right]+\frac{\left|z_{1}\right|^{2} \nabla_{1}}{\|z\|^{2}}\left[q\left(z_{1}\right)\right. \\
& \left.-\frac{1-c^{2}}{2 c} \frac{-\alpha+i \tan \beta}{1-\alpha}+\frac{1+c^{2}}{2 c}\right]\left[1+\frac{1}{f} \sum_{j=2}^{\infty}\left(\frac{f}{z_{1}}\right)^{\gamma j}\right. \\
& \left.\cdot(1-j) P_{j}\left(z_{0}\right)\right]+\frac{\left\|z_{0}\right\|^{2} \nabla_{1}}{\|z\|^{2}}\left[\frac{1-c^{2}}{2 c} \frac{1-i \tan \beta}{1-\alpha}\right. \\
& \left.+\gamma\left(q\left(z_{1}\right)+c\right)\left(1+\frac{1}{f} \sum_{j=2}^{\infty}\left(\frac{f}{z_{1}}\right)^{\gamma j}(1-j) P_{j}\left(z_{0}\right)\right)\right] \\
& +\left(\frac{1-c^{2}}{2 c} \frac{-\alpha+i \tan \beta}{1-\alpha}-\frac{1+c^{2}}{2 c}\right)\left(\nabla_{1}+\nabla_{2}\right) \\
& =-c\left(\frac{\left\|z_{0}\right\|^{2} \nabla_{1}}{\|z\|^{2}}+\nabla_{2}\right)+\left[\widetilde{\nabla_{2}}\left(q\left(z_{1}\right)+c\right)+\frac{\left|z_{1}\right|^{2}}{\|z\|^{2}}\right. \\
& \left.\cdot \nabla_{1} q\left(z_{1}\right)+\frac{\gamma\left\|z_{0}\right\|^{2}}{\|z\|^{2}} \nabla_{1}\left(q\left(z_{1}\right)+c\right)\right]\left[1+\frac{1}{f}\right. \\
& \left.\cdot \sum_{j=2}^{\infty}\left(\frac{f}{z_{1}}\right)^{\gamma j}(1-j) P_{j}\left(z_{0}\right)\right]+\frac{\left|z_{1}\right|^{2}}{\|z\|^{2}} \nabla_{1}\left(\frac{1+c^{2}}{2 c}\right. \\
& \left.-\frac{1-c^{2}}{2 c} \frac{-\alpha+i \tan \beta}{1-\alpha}\right) \frac{1}{f} \sum_{j=2}^{\infty}\left(\frac{f}{z_{1}}\right)^{\gamma j}(1-j) P_{j}\left(z_{0}\right)
\end{aligned}
$$

$$
\begin{aligned}
& =c(\gamma-1) \frac{\left\|z_{0}\right\|^{2} \nabla_{1}}{\|z\|^{2}}+c\left(\widetilde{\nabla_{2}}-\nabla_{2}\right)+\left(\widetilde{\nabla_{2}}\right. \\
& \left.+\frac{\left|z_{1}\right|^{2}+\gamma\left\|z_{0}\right\|^{2}}{\|z\|^{2}} \nabla_{1}\right) q\left(z_{1}\right)+\left(\widetilde{\nabla_{2}}\right. \\
& \left.+\frac{\left|z_{1}\right|^{2}+\gamma\left\|z_{0}\right\|^{2}}{\|z\|^{2}} \nabla_{1}\right)\left(q\left(z_{1}\right)+c\right) \frac{1}{f} \sum_{j=2}^{\infty}\left(\frac{f}{z_{1}}\right)^{\gamma j}(1 \\
& -j) P_{j}\left(z_{0}\right)+\frac{1-c^{2}}{2 c} \frac{1-i \tan \beta}{1-\alpha} \frac{\left|z_{1}\right|^{2}}{\|z\|^{2}} \nabla_{1} \frac{1}{f} \\
& \cdot \sum_{j=2}^{\infty}\left(\frac{f}{z_{1}}\right)^{\gamma j}(1-j) P_{j}\left(z_{0}\right),
\end{aligned}
$$

where $\widetilde{\nabla_{2}}=\sum_{k=1}^{s} \delta_{k} p_{k}\left\|w_{k}\right\|^{2 p_{k}}$. We use (26) and $\left|h\left(z_{1}\right)\right|<1$ to see that

$$
\begin{aligned}
& \left(\nabla_{1}+\nabla_{2}\right)(|I|-1)<c(1-\gamma) \frac{\left\|z_{0}\right\|^{2} \nabla_{1}}{\|z\|^{2}}+c\left(\nabla_{2}-\widetilde{\nabla_{2}}\right) \\
& +\widetilde{\nabla_{2}}+\frac{\left|z_{1}\right|^{2}+\gamma\left\|z_{0}\right\|^{2}}{\|z\|^{2}} \nabla_{1}+\frac{1-c^{2}}{2 c}\left(\widetilde{\nabla_{2}}\right. \\
& \left.+\frac{\left|z_{1}\right|^{2}+\gamma\left\|z_{0}\right\|^{2}}{\|z\|^{2}} \nabla_{1}\right) \frac{\left|z_{1}\right|}{|f|} \sum_{j=2}^{n}\left|\frac{f}{z_{1}}\right|^{\gamma j}(j-1)\left|P_{j}\left(z_{0}\right)\right| \\
& +\frac{1-c^{2}}{2 c} \frac{1}{(1-\alpha) \cos \beta} \frac{\left|z_{1}\right|^{2}}{\|z\|^{2}} \nabla_{1} \frac{1}{|f|} \sum_{j=2}^{\infty}\left(\frac{f}{z_{1}}\right)^{\gamma j}(j \\
& -1)\left|P_{j}\left(z_{0}\right)\right|-\left(\nabla_{1}+\nabla_{2}\right) \leq(c-1)(1-\gamma) \frac{\left\|z_{0}\right\|^{2} \nabla_{1}}{\|z\|^{2}} \\
& +(c-1)\left(\nabla_{2}-\widetilde{\nabla_{2}}\right)+\frac{\nabla_{1}}{\|z\|^{2}} \\
& +\frac{1-c^{2}}{2 c}\left[\left(\frac{\widetilde{\nabla_{2}}}{\nabla_{1}}\|z\|^{2}+\left|z_{1}\right|^{2}+\gamma\left\|z_{0}\right\|^{2}\right)\right. \\
& +\frac{1-\alpha) \cos \beta}{|f|} \sum_{j=2}^{\infty}\left|\frac{f}{z_{1}}\right|^{\gamma j}(j-1)\left|P_{j}\left(z_{0}\right)\right| .
\end{aligned}
$$

Note that $(w, z) \in \Omega_{p_{1}, \ldots, p_{s}, q}^{B_{n}}$ implies that

$$
\begin{aligned}
& \sum_{k=1}^{s}\left\|w_{(k)}\right\|^{2 p_{k}}<K_{B^{n}}(z, z)^{-q} \\
& \quad=\left(\frac{\pi^{n}}{n !}\right)^{q}\left(1-\|z\|^{2}\right)^{(n+1) q}, \\
& \nabla_{1}=(n+1) q \pi^{n q}(n !)^{-q}\left(1-\|z\|^{2}\right)^{(n+1) q-1}\|z\|^{2}, \\
& \widetilde{\nabla_{2}}<\delta \sum_{k=1}^{s}\left\|w_{k}\right\|^{2 p_{k}}
\end{aligned}
$$


lead to

$$
\frac{\widetilde{\nabla_{2}}}{\nabla_{1}}<\frac{\delta\left(1-\|z\|^{2}\right)}{(n+1) q\|z\|^{2}} .
$$

Therefore

$$
\begin{aligned}
& \frac{\widetilde{\nabla_{2}}}{\nabla_{1}}\|z\|^{2}+\left|z_{1}\right|^{2}+\gamma\left\|z_{0}\right\|^{2} \\
& <\frac{\delta}{(n+1) q}\left(1-\|z\|^{2}\right)+\|z\|^{2}+(\gamma-1)\left\|z_{0}\right\|^{2} \\
& =\frac{\delta}{(n+1) q}+\left(1-\frac{\delta}{(n+1) q}\right)\|z\|^{2} \\
& \quad+(\gamma-1)\left\|z_{0}\right\|^{2}<1
\end{aligned}
$$

provided that $q \geq \delta /(n+1)$.

Lemma 7 and (38) eventually lead us to the following inequality string:

$$
\begin{aligned}
\left(\nabla_{1}+\nabla_{2}\right)(|I|-1)<(c-1)(1-\gamma) \frac{\left\|z_{0}\right\|^{2} \nabla_{1}}{\|z\|^{2}}+\frac{\nabla_{1}}{\|z\|^{2}} \\
\cdot \frac{1-c^{2}}{2 c} \frac{1+(1-\alpha) \cos \beta}{(1-\alpha) \cos \beta} \frac{\left|z_{1}\right|}{|f|} \sum_{j=2}^{\infty}\left|\frac{f}{z_{1}}\right|^{\gamma j}(j-1) \\
\cdot\left|P_{j}\left(z_{0}\right)\right| \leq(c-1)(1-\gamma) \frac{\left\|z_{0}\right\|^{2} \nabla_{1}}{\|z\|^{2}}+\frac{\nabla_{1}}{\|z\|^{2}} \frac{1-c^{2}}{2 c} \\
\cdot \frac{1+(1-\alpha) \cos \beta}{(1-\alpha) \cos \beta}\left(1+\left|z_{1}\right|\right)^{2} \\
\cdot \sum_{j=2}^{\infty}\left(1-\left|z_{1}\right|\right)^{-2 \gamma j}(j-1)\left\|P_{j}\right\|\left\|z_{0}\right\|^{j}=\frac{1-c^{2}}{2 c} \\
\cdot \frac{1+(1-\alpha) \cos \beta}{(1-\alpha) \cos \beta} \frac{\left\|z_{0}\right\|^{2} \nabla_{1}}{\|z\|^{2}}\left[\sum_{j=2}^{\infty}\left(1+\left|z_{1}\right|\right)^{j / 2+1}\right. \\
\left.\cdot \frac{\left(1-\left|z_{1}\right|\right)^{j / 2-2 \gamma j-1}(j-1)\left\|P_{j}\right\|}{(1+c)(1+(1-\alpha) \cos \beta)}\right] \leq 0, \\
-\frac{1+(1-\alpha) \cos \beta}{(1+c)(1-\gamma)(1-\alpha) \cos \beta} \frac{\left\|z_{0}\right\|^{2} \nabla_{1}}{\|z\|^{2}}\left[\sum_{j=2}^{\infty} 2^{j / 2+1}(j-1)\left\|P_{j}\right\|\right. \\
(1-\alpha) \cos \beta
\end{aligned}
$$

where $P_{j}=0$ for $j<2 /(1-4 \gamma)$ and

$$
\sum_{j=2}^{\infty} 2^{j / 2+1}(j-1)\left\|P_{j}\right\| \leq \frac{2 c(1-\gamma)(1-\alpha) \cos \beta}{(1+c)(1+(1-\alpha) \cos \beta)} .
$$

This completes the proof.
Setting $w_{(1)}=\cdots=w_{(s)}=0$ in Theorem 11, the operator (4) reduces to the generalized Roper-Suffridge operator (6) and the Bergman-Hartogs domain $\Omega$ reduces to the unit ball $B^{n}$; thus, we draw the following conclusion on $B^{n}$ in $\mathbb{C}^{n}$.

Corollary 12. Let $f\left(z_{1}\right)$ be a strong and almost spiral-like function of type $\beta$ and order $\alpha$ on $D$ with $\alpha \in[0,1)$ and $\beta \in(-\pi / 2, \pi / 2), c \in(0,1)$. Let $F(z)$ be the mapping denoted by (6) with $\gamma<1 / 4$ and $\left.\left(f\left(z_{1}\right) / z_{1}\right)^{\gamma}\right|_{z_{1}=0}=1$. Then $F(z)$ is a strong and almost spiral-like mapping of type $\beta$ and order $\alpha$ on $B^{n}$ provided that $P_{j}=0$ for $j<2 /(1-4 \gamma)$ and

$$
\sum_{j=2}^{\infty} 2^{j / 2+1}(j-1)\left\|P_{j}\right\| \leq \frac{2 c(1-\gamma)(1-\alpha) \cos \beta}{(1+c)(1+(1-\alpha) \cos \beta)} .
$$

Remark 13. Setting $\alpha=0$ and $\beta=0$ in Theorem 11 and Corollary 12, respectively, we get the corresponding results for strong and almost star-like mappings of order $\alpha$ and strong spiral-like mapping of type $\beta$.

If we have the precise growth result of strong and almost spiral-like mapping of type $\beta$ and order $\alpha$ and apply the growth result in Theorem 11, we can get more precise conclusion. But, up to now we only have the growth theorem of strong spiral-like function of type $\beta$ [22]. In the following, we apply it in the process of discussing the invariance of strong spiral-like function of type $\beta$.

Theorem 14. Let $f\left(z_{1}\right)$ be a strong spiral-like function of type $\beta$ on $D$ with $\beta \in(-\pi / 2, \pi / 2)$ and $c \in(0,1)$. Let $F(w, z)$ be the mapping denoted by (4) with $p_{i}>1, \delta_{i} \in$ $[0,1](i=1, \ldots, s), \gamma<1 / 4$ and $\left.\left(f\left(z_{1}\right) / z_{1}\right)^{\delta_{i}}\right|_{z_{1}=0}=$ $1,\left.\left(f\left(z_{1}\right) / z_{1}\right)^{\gamma}\right|_{z_{1}=0}=1$. Then $F(w, z)$ is a strong spiral-like mapping of type $\beta$ and order $\alpha$ on $\Omega$ provided that $P_{j}=0$ for $j<2 /(1-4 \gamma)$ and

$$
\begin{aligned}
q & \geq \frac{\delta}{n+1}, \\
\sum_{j=2}^{\infty} 2^{j / 2-1}(j-1)\left\|P_{j}\right\| & \leq \frac{2 c(1-\gamma) \cos \beta}{(1+c)^{3}(1+\cos \beta)},
\end{aligned}
$$

where $\delta=\max \left\{p_{1} \delta_{1}, \ldots, p_{s} \delta_{s}\right\}$.

Proof. Setting $\alpha=0$ in (39) and applying Lemma 8 we obtain the following inequality string:

$$
\begin{gathered}
\left(\nabla_{1}+\nabla_{2}\right)(|I|-1)<(c-1)(1-\gamma) \frac{\left\|z_{0}\right\|^{2} \nabla_{1}}{\|z\|^{2}}+\frac{\nabla_{1}}{\|z\|^{2}} \\
\cdot \frac{1-c^{2}}{2 c} \frac{1+\cos \beta}{\cos \beta} \frac{\left|z_{1}\right|}{|f|} \sum_{j=2}^{\infty}\left|\frac{f}{z_{1}}\right|^{\gamma j}(j-1)\left|P_{j}\left(z_{0}\right)\right| \\
\leq(c-1)(1-\gamma) \frac{\left\|z_{0}\right\|^{2} \nabla_{1}}{\|z\|^{2}}+\frac{\nabla_{1}}{\|z\|^{2}} \frac{1-c^{2}}{2 c} \\
\cdot \frac{1+\cos \beta}{\cos \beta}\left(1+c\left|z_{1}\right|\right)^{2} \sum_{j=2}^{\infty}\left(1-c\left|z_{1}\right|\right)^{-2 \gamma j}(j-1)
\end{gathered}
$$




$$
\begin{aligned}
& \cdot\left\|P_{j}\right\|\left\|z_{0}\right\|^{j} \leq \frac{1-c^{2}}{2 c} \frac{1+\cos \beta}{\cos \beta} \\
& \cdot \frac{\left\|z_{0}\right\|^{2} \nabla_{1}}{\|z\|^{2}}\left[\sum_{j=2}^{\infty}\left(1+c\left|z_{1}\right|\right)^{2}\left(1+\left|z_{1}\right|\right)^{j / 2-1}\right. \\
& \cdot\left(1-c\left|z_{1}\right|\right)^{j / 2-2 \gamma j-1}(j-1)\left\|P_{j}\right\| \\
& \left.-\frac{2 c(1-\gamma) \cos \beta}{(1+c)(1+\cos \beta)}\right] \leq \frac{1-c^{2}}{2 c} \frac{1+\cos \beta}{\cos \beta} \\
& \cdot \frac{\left\|z_{0}\right\|^{2} \nabla_{1}}{\|z\|^{2}}\left[\sum_{j=2}^{\infty}(1+c)^{2} 2^{j / 2-1}(j-1)\left\|P_{j}\right\|\right. \\
& \left.-\frac{2 c(1-\gamma) \cos \beta}{(1+c)(1+\cos \beta)}\right] \leq 0,
\end{aligned}
$$

where $P_{j}=0$ for $j<2 /(1-4 \gamma)$ and

$$
\sum_{j=2}^{\infty} 2^{j / 2-1}(j-1)\left\|P_{j}\right\| \leq \frac{2 c(1-\gamma) \cos \beta}{(1+c)^{3}(1+\cos \beta)} .
$$

Hence the assertion follows.

\section{The Invariance of $S_{\Omega}^{*}(\beta, A, B)$}

As above, write $\Omega_{p_{1}, \ldots, p_{s}, q}^{B^{n}}=\Omega$. In this section, we mainly study the perturbed Extension Operator (4) preserving the geometric characteristics of $S_{\Omega}^{*}(\beta, A, B)$ on $\Omega$ and then generalize the conclusion on $B^{n}$ and get the invariance of some subclasses.

Theorem 15. Let $f\left(z_{1}\right) \in S_{D}^{*}(\beta, A, B)$ with $-1 \leq A<B<$ $(A+1) / 2<1$ and $\beta \in(-\pi / 2, \pi / 2)$. Let $F(w, z)$ be the mapping denoted by (4) with $p_{i}>1, \delta_{i} \in[0,1](i=1, \ldots, s), \gamma \in$ $(0,1 / 4)$ and $\left.\left(f\left(z_{1}\right) / z_{1}\right)^{\delta_{i}}\right|_{z_{1}=0}=1,\left.\left(f\left(z_{1}\right) / z_{1}\right)^{\gamma}\right|_{z_{1}=0}=1$. Then $F(w, z) \in S_{\Omega}^{*}(\beta, A, B)$ provided that $P_{j}=0$ for $j<2 /(1-4 \gamma)$ and

$$
\begin{aligned}
q & \geq \frac{\delta}{n+1}, \\
\sum_{j=2}^{\infty} 2^{j / 2+1}(j-1)\left\|P_{j}\right\| & \leq \frac{(1-\gamma)(B-A) \cos \beta}{(1+|B|)(1+\cos \beta)},
\end{aligned}
$$

where $\delta=\max \left\{p_{1} \delta_{1}, \ldots, p_{s} \delta_{s}\right\}$.

Proof. By Definition 2, we need to prove

$$
\begin{aligned}
& \mid \frac{1-B^{2}}{B-A}\left[i \tan \beta+(1-i \tan \beta) \frac{2}{\rho(w, z)}\right. \\
& \left.\quad \cdot \frac{\partial \rho(w, z)}{\partial(w, z)}(D F(w, z))^{-1} F(w, z)\right]-\frac{1-A B}{B-A} \mid<1 .
\end{aligned}
$$

Similar to Theorem 11, we need only to prove that (46) holds for $(w, z) \in \partial \Omega$ which implies $\rho(w, z)=1$.

Since $f\left(z_{1}\right) \in S_{D}^{*}(\beta, A, B)$, by Definition 2 , we have

$$
\begin{aligned}
& \left|i \tan \beta+(1-i \tan \beta) \frac{f\left(z_{1}\right)}{z_{1} f^{\prime}\left(z_{1}\right)}-\frac{1-A B}{1-B^{2}}\right| \\
& \quad<\frac{B-A}{1-B^{2}} .
\end{aligned}
$$

Let

$$
\begin{aligned}
& \frac{1-B^{2}}{B-A} i \tan \beta+\frac{1-B^{2}}{B-A}(1-i \tan \beta) \frac{f\left(z_{1}\right)}{z_{1} f^{\prime}\left(z_{1}\right)} \\
& -\frac{1-A B}{B-A}=h\left(z_{1}\right) .
\end{aligned}
$$

Then $\left|h\left(z_{1}\right)\right|<1$ and $h(0)=-B$. Letting $g\left(z_{1}\right)=(B-A) /(1-$ $\left.B^{2}\right)\left(h\left(z_{1}\right)+B\right)$, we get $g\left(z_{1}\right) \in H(D), \quad g(0)=0$ and $\left|h\left(z_{1}\right)\right|=$ $\left|\left(\left(1-B^{2}\right) /(B-A)\right) g\left(z_{1}\right)-B\right|<1$. In view of $\left(\left(1-B^{2}\right) /(B-\right.$ A) $)\left|g\left(z_{1}\right)\right|-|B| \leq\left|\left(\left(1-B^{2}\right) /(B-A)\right) g\left(z_{1}\right)-B\right|$, we obtain $\left|g\left(z_{1}\right)\right|<(1+|B|)(B-A) /\left(1-B^{2}\right) \leq 1$ where $-1 \leq A<B<$ $(A+1) / 2<1$. By Schwarz Lemma, we get $\left|g\left(z_{1}\right)\right| \leq\left|z_{1}\right|$ which implies that

$$
\left|h\left(z_{1}\right)+B\right| \leq \frac{1-B^{2}}{B-A}\left|z_{1}\right|
$$

Let

$$
\begin{aligned}
& \frac{1-B^{2}}{B-A} i \tan \beta+\frac{1-B^{2}}{B-A}(1-i \tan \beta) \\
& \quad \cdot \frac{2 \partial \rho(w, z)}{\partial(w, z)}(D F(w, z))^{-1} F(w, z)-\frac{1-A B}{B-A}=I .
\end{aligned}
$$

Then, (31) and (48) lead to the following equation string:

$$
\begin{aligned}
& \left(\nabla_{1}+\nabla_{2}\right) I=\frac{1-B^{2}}{B-A}(1-i \tan \beta)\left\{\sum_{k=1}^{s} p_{k}\left\|w_{(k)}\right\|^{2 p_{k}}[1\right. \\
& -\delta_{k} \\
& \left.. \frac{z_{1} f^{\prime}-f}{z_{1} f^{\prime}}\left(1+\frac{1}{f} \sum_{j=2}^{\infty}\left(\frac{f}{z_{1}}\right)^{\gamma j}(1-j) P_{j}\left(z_{0}\right)\right)\right] \\
& +\frac{\left|z_{1}\right|^{2} \nabla_{1}}{\|z\|^{2}} \frac{f}{z_{1} f^{\prime}}\left[1+\frac{1}{f} \sum_{j=2}^{\infty}\left(\frac{f}{z_{1}}\right)^{\gamma j}(1-j) P_{j}\left(z_{0}\right)\right] \\
& +\left(\frac{1-B^{2}}{B-A} i \tan \beta-\frac{1-A B}{B-A}\right)^{\prime}\left(\nabla_{1}+\nabla_{2}\right) \\
& +\frac{\left\|z_{0}\right\|^{2} \nabla_{1}}{\|z\|^{2}}[1-\gamma \\
& \left.\left.+\frac{z_{1} f^{\prime}-f}{z_{1} f^{\prime}}\left(1+\frac{1}{f} \sum_{j=2}^{\infty}\left(\frac{f}{z_{1}}\right)^{\gamma j}(1-j) P_{j}\left(z_{0}\right)\right)\right]\right\}
\end{aligned}
$$




$$
\begin{aligned}
& =\frac{1-B^{2}}{B-A}(1-i \tan \beta) \nabla_{2}+\widetilde{\nabla_{2}}\left[h\left(z_{1}\right)+B\right]\left[1+\frac{1}{f}\right. \\
& \left.\cdot \sum_{j=2}^{\infty}\left(\frac{f}{z_{1}}\right)^{\gamma j}(1-j) P_{j}\left(z_{0}\right)\right]+\frac{\left|z_{1}\right|^{2} \nabla_{1}}{\|z\|^{2}}\left[h\left(z_{1}\right)\right. \\
& \left.-\frac{1-B^{2}}{B-A} i \tan \beta+\frac{1-A B}{B-A}\right]\left[1+\frac{1}{f} \sum_{j=2}^{\infty}\left(\frac{f}{z_{1}}\right)^{\gamma j}\right. \\
& \left.\cdot(1-j) P_{j}\left(z_{0}\right)\right]+\frac{\left\|z_{0}\right\|^{2} \nabla_{1}}{\|z\|^{2}}\left[\frac{1-B^{2}}{B-A}(1-i \tan \beta)\right. \\
& +\gamma\left(h\left(z_{1}\right)+B\right)\left(1+\frac{1}{f}\right. \\
& \left.\left.\cdot \sum_{j=2}^{\infty}\left(\frac{f}{z_{1}}\right)^{\gamma j}(1-j) P_{j}\left(z_{0}\right)\right)\right]+\left(\frac{1-B^{2}}{B-A} i \tan \beta\right. \\
& \left.-\frac{1-A B}{B-A}\right)\left(\nabla_{1}+\nabla_{2}\right)=B(\gamma-1) \frac{\left\|z_{0}\right\|^{2} \nabla_{1}}{\|z\|^{2}}+B\left(\widetilde{\nabla_{2}}\right. \\
& \left.-\nabla_{2}\right)+\left[\widetilde{\nabla_{2}}+\frac{\nabla_{1}}{\|z\|^{2}}\left(\left|z_{1}\right|^{2}+\gamma\left\|z_{0}\right\|^{2}\right)\right] h\left(z_{1}\right) \\
& +\left[\widetilde{\nabla_{2}}\left(h\left(z_{1}\right)+B\right)+\frac{\left|z_{1}\right|^{2} \nabla_{1}}{\|z\|^{2}}\left(h\left(z_{1}\right)-\frac{1-B^{2}}{B-A} i\right.\right. \\
& \left.\left.\cdot \tan \beta+\frac{1-A B}{B-A}\right)+\frac{\gamma\left\|z_{0}\right\|^{2} \nabla_{1}}{\|z\|^{2}}\left(h\left(z_{1}\right)+B\right)\right] \frac{1}{f} \\
& \cdot \sum_{j=2}^{\infty}\left(\frac{f}{z_{1}}\right)^{\gamma j}(1-j) P_{j}\left(z_{0}\right)=B(\gamma-1) \frac{\left\|z_{0}\right\|^{2}}{\|z\|^{2}} \nabla_{1} \\
& +B\left(\widetilde{\nabla_{2}}-\nabla_{2}\right)+\left[\widetilde{\nabla_{2}}+\frac{\nabla_{1}}{\|z\|^{2}}\left(\left|z_{1}\right|^{2}+\gamma\left\|z_{0}\right\|^{2}\right)\right] \\
& \cdot h\left(z_{1}\right)+\left[\widetilde{\nabla_{2}}+\frac{\nabla_{1}}{\|z\|^{2}}\left(\left|z_{1}\right|^{2}+\gamma\left\|z_{0}\right\|^{2}\right)\right]\left(h\left(z_{1}\right)\right. \\
& \text { +B) } \frac{1}{f} \sum_{j=2}^{\infty}\left(\frac{f}{z_{1}}\right)^{\gamma j}(1-j) P_{j}\left(z_{0}\right)+\frac{1-B^{2}}{B-A}(1-i \\
& \cdot \tan \beta) \frac{\left|z_{1}\right|^{2} \nabla_{1}}{\|z\|^{2}} \frac{1}{f} \sum_{j=2}^{\infty}\left(\frac{f}{z_{1}}\right)^{\gamma j}(1-j) P_{j}\left(z_{0}\right) .
\end{aligned}
$$

By (49), (38), and Lemma 7 there comes the following inequality string:

$$
\begin{aligned}
& \left(\nabla_{1}+\nabla_{2}\right)(|I|-1)<|B|(1-\gamma) \frac{\left\|z_{0}\right\|^{2}}{\|z\|^{2}} \nabla_{1}+|B|\left(\nabla_{2}\right. \\
& \left.-\widetilde{\nabla_{2}}\right)+\left[\widetilde{\nabla_{2}}+\frac{\nabla_{1}}{\|z\|^{2}}\left(\left|z_{1}\right|^{2}+\gamma\left\|z_{0}\right\|^{2}\right)\right]-\left(\nabla_{1}+\nabla_{2}\right)
\end{aligned}
$$

$+\frac{1-B^{2}}{B-A}\left[\widetilde{\nabla_{2}}+\frac{\nabla_{1}}{\|z\|^{2}}\left(\left|z_{1}\right|^{2}+\gamma\left\|z_{0}\right\|^{2}\right)\right] \mid \frac{z_{1}}{f}$

$\cdot \sum_{j=2}^{\infty}\left|\frac{f}{z_{1}}\right|^{\gamma j}(j-1)\left|P_{j}\left(z_{0}\right)\right|+\frac{1-B^{2}}{(B-A) \cos \beta} \frac{\left|z_{1}\right| \nabla_{1}}{\|z\|^{2}}$

$\cdot \frac{1}{|f|} \sum_{j=2}^{\infty}\left|\frac{f}{z_{1}}\right|^{\gamma j}(j-1)\left|P_{j}\left(z_{0}\right)\right|=(|B|-1)(1-\gamma)$

$\frac{\left\|z_{0}\right\|^{2}}{\|z\|^{2}} \nabla_{1}+(|B|-1)\left(\nabla_{2}-\widetilde{\nabla_{2}}\right)+\frac{1-B^{2}}{B-A}$

$\cdot \frac{\nabla_{1}}{\|z\|^{2}}\left[\frac{\widetilde{\nabla_{2}}}{\nabla_{1}}\|z\|^{2}+\left|z_{1}\right|^{2}+\gamma\left\|z_{0}\right\|^{2}+\frac{1}{\cos \beta}\right]\left|\frac{z_{1}}{f}\right|$

$\cdot \sum_{j=2}^{\infty}\left|\frac{f}{z_{1}}\right|^{\gamma j}(j-1)\left|P_{j}\left(z_{0}\right)\right|<(|B|-1)(1-\gamma) \frac{\left\|z_{0}\right\|^{2}}{\|z\|^{2}}$

$\cdot \nabla_{1}+\frac{1-B^{2}}{B-A} \frac{1+\cos \beta}{\cos \beta} \frac{\nabla_{1}}{\|z\|^{2}}\left|\frac{z_{1}}{f}\right| \sum_{j=2}^{\infty}\left|\frac{f}{z_{1}}\right|^{\gamma j}(j-1)$

$\cdot\left\|P_{j}\right\|\left\|z_{0}\right\|^{j}=\frac{1-B^{2}}{B-A} \frac{1+\cos \beta}{\cos \beta} \frac{\left\|z_{0}\right\|^{2} \nabla_{1}}{\|z\|^{2}}\left[\left|\frac{z_{1}}{f}\right|\right.$

$\cdot \sum_{j=2}^{\infty}\left|\frac{f}{z_{1}}\right|^{\gamma j}(j-1)\left\|P_{j}\right\|\left\|z_{0}\right\|^{j-2}$

$\left.-\frac{(1-\gamma)(B-A) \cos \beta}{(1+|B|)(1+\cos \beta)}\right] \leq \frac{1-B^{2}}{B-A} \frac{1+\cos \beta}{\cos \beta}$

$\cdot \frac{\left\|z_{0}\right\|^{2} \nabla_{1}}{\|z\|^{2}}\left[\sum_{j=2}^{\infty}\left(1+\left|z_{1}\right|\right)^{j / 2+1}\left(1-\left|z_{1}\right|\right)^{j / 2-2 \gamma j-1}\right.$

$\left.\cdot(j-1)\left\|P_{j}\right\|-\frac{(1-\gamma)(B-A) \cos \beta}{(1+|B|)(1+\cos \beta)}\right] \leq \frac{1-B^{2}}{B-A}$

$\cdot \frac{1+\cos \beta}{\cos \beta} \frac{\left\|z_{0}\right\|^{2} \nabla_{1}}{\|z\|^{2}}\left[\sum_{j=2}^{\infty} 2^{j / 2+1}(j-1)\left\|P_{j}\right\|\right.$

$\left.-\frac{(1-\gamma)(B-A) \cos \beta}{(1+|B|)(1+\cos \beta)}\right] \leq 0$,

where $P_{j}=0$ for $j<2 /(1-4 \gamma)$ and

$$
\sum_{j=2}^{\infty} 2^{j / 2+1}(j-1)\left\|P_{j}\right\| \leq \frac{(1-\gamma)(B-A) \cos \beta}{(1+|B|)(1+\cos \beta)} .
$$

Therefore (46) holds, which follows $F(w, z) \in S_{\Omega}^{*}(\beta, A, B)$.

Setting $w_{(1)}=\cdots=w_{(s)}=0$ in Theorem 15 , we draw the following invariance of the mappings $S_{B^{n}}^{*}(\beta, A, B)$ under the 
generalized Roper-Suffridge operator (6) on the unit ball $B^{n}$ in $\mathbb{C}^{n}$.

Corollary 16. Let $f\left(z_{1}\right) \in S_{D}^{*}(\beta, A, B)$ with $-1 \leq A<B<$ $(A+1) / 2<1$ and $\beta \in(-\pi / 2, \pi / 2)$. Let $F(z)$ be the mapping denoted by (6) with $\gamma \in(0,1 / 4)$ and $\left.\left(f\left(z_{1}\right) / z_{1}\right)^{\gamma}\right|_{z_{1}=0}=1$. Then $F(z) \in S_{B^{n}}^{*}(\beta, A, B)$ provided that $P_{j}=0$ for $j<2 /(1-$ $4 \gamma$ ) and

$$
\sum_{j=2}^{\infty} 2^{j / 2+1}(j-1)\left\|P_{j}\right\| \leq \frac{(1-\gamma)(B-A) \cos \beta}{(1+|B|)(1+\cos \beta)} .
$$

In the process of discussing the invariance of $S_{\Omega}^{*}(A, B)$, if we apply the growth theorem of $S_{\Omega}^{*}(A, B)$ in [23], we can get the following more precise conclusion.

Theorem 17. Let $f\left(z_{1}\right) \in S_{D}^{*}(A, B)$ with $-1 \leq A<B<$ $(A+1) / 2<1$. Let $F(w, z)$ be the mapping denoted by (4) with $p_{i}>1, \delta_{i} \in[0,1](i=1, \ldots, s), \gamma \in(0,1 / 4)$ and $\left.\left(f\left(z_{1}\right) / z_{1}\right)^{\delta_{i}}\right|_{z_{1}=0}=1,\left.\left(f\left(z_{1}\right) / z_{1}\right)^{\gamma}\right|_{z_{1}=0}=1$. Let $q \geq \delta /(n+1)$ where $\delta=\max \left\{p_{1} \delta_{1}, \ldots, p_{s} \delta_{s}\right\}$. Then we have the following conclusions:

(1) For $A \neq 0, F(w, z) \in S_{\Omega}^{*}(A, B)$ provided that

$$
\begin{aligned}
& \sum_{j=2}^{\infty}(1-A)^{(A-B) / A}(1+A)^{((B-A) / A) \gamma j}(j-1)\left\|P_{j}\right\| \\
& \quad \leq \frac{(1-\gamma)(B-A)}{2(1+|B|)} .
\end{aligned}
$$

(2) For $A=0$ and $B>0, F(w, z) \in S_{\Omega}^{*}(A, B)$ provided that

$$
\sum_{j=2}^{\infty} e^{B(1+\gamma j)}(j-1)\left\|P_{j}\right\| \leq \frac{(1-\gamma)(B-A)}{2(1+|B|)} .
$$

(3) For $A=0$ and $B<0, F(w, z) \in S_{\Omega}^{*}(A, B)$ provided that

$$
\sum_{j=2}^{\infty}(j-1)\left\|P_{j}\right\| \leq \frac{(1-\gamma)(B-A)}{2(1+|B|)} .
$$

Proof. Setting $\beta=0$ in the proof of Theorem 15, by using (52) and Lemma 9 we obtain the following conclusions:

(1) For the case that $A \neq 0$ we get

$$
\begin{gathered}
\left(\nabla_{1}+\nabla_{2}\right)(|I|-1)<\frac{1-B^{2}}{B-A} \frac{1+\cos \beta}{\cos \beta} \frac{\left\|z_{0}\right\|^{2} \nabla_{1}}{\|z\|^{2}}\left[\left|\frac{z_{1}}{f}\right|\right. \\
\cdot \sum_{j=2}^{\infty}\left|\frac{f}{z_{1}}\right|^{\gamma j}(j-1)\left\|P_{j}\right\|\left\|z_{0}\right\|^{j-2} \\
\left.-\frac{(1-\gamma)(B-A) \cos \beta}{(1+|B|)(1+\cos \beta)}\right] \leq \frac{1-B^{2}}{B-A} \frac{1+\cos \beta}{\cos \beta} \\
\cdot \frac{\left\|z_{0}\right\|^{2} \nabla_{1}}{\|z\|^{2}}\left[\sum_{j=2}^{\infty}\left(1-A\left|z_{1}\right|\right)^{(A-B) / A}\right.
\end{gathered}
$$

$$
\begin{aligned}
& \cdot\left(1+A\left|z_{1}\right|\right)^{((B-A) / A) \gamma j}\left(1-\left|z_{1}\right|^{2}\right)^{j / 2-1}(j-1)\left\|P_{j}\right\| \\
& \left.-\frac{(1-\gamma)(B-A)}{2(1+|B|)}\right] \leq \frac{1-B^{2}}{B-A} \frac{1+\cos \beta}{\cos \beta} \\
& \cdot \frac{\left\|z_{0}\right\|^{2} \nabla_{1}}{\|z\|^{2}}\left[\sum_{j=2}^{\infty}(1-A)^{(A-B) / A}(1+A)^{((B-A) / A) \gamma j}\right. \\
& \left.\cdot(j-1)\left\|P_{j}\right\|-\frac{(1-\gamma)(B-A)}{2(1+|B|)}\right] \leq 0,
\end{aligned}
$$

where

$$
\begin{aligned}
& \sum_{j=2}^{\infty}(1-A)^{(A-B) / A}(1+A)^{((B-A) / A) \gamma j}(j-1)\left\|P_{j}\right\| \\
& \quad \leq \frac{(1-\gamma)(B-A)}{2(1+|B|)} .
\end{aligned}
$$

(2) For the case that $A=0$ and $B>0$ we have

$$
\begin{aligned}
& \left(\nabla_{1}+\nabla_{2}\right)(|I|-1)<\frac{1-B^{2}}{B-A} \frac{1+\cos \beta}{\cos \beta} \frac{\left\|z_{0}\right\|^{2} \nabla_{1}}{\|z\|^{2}}\left[\left|\frac{z_{1}}{f}\right|\right. \\
& \cdot \sum_{j=2}^{\infty}\left|\frac{f}{z_{1}}\right|^{\gamma j}(j-1)\left\|P_{j}\right\|\left\|z_{0}\right\|^{j-2} \\
& \left.-\frac{(1-\gamma)(B-A) \cos \beta}{(1+|B|)(1+\cos \beta)}\right] \leq \frac{1-B^{2}}{B-A} \frac{1+\cos \beta}{\cos \beta} \\
& \cdot \frac{\left\|z_{0}\right\|^{2} \nabla_{1}\left[\sum_{j=2}^{\infty} e^{B\left|z_{1}\right|(1+\gamma j)}\left(1-\left|z_{1}\right|^{2}\right)^{j / 2-1}(j-1)\right.}{\|z\|^{2}} \\
& \left.\cdot\left\|P_{j}\right\|^{-} \frac{(1-\gamma)(B-A)}{2(1+|B|)}\right] \leq \frac{1-B^{2}}{B-A} \frac{1+\cos \beta}{\cos \beta} \\
& \cdot \frac{\left\|z_{0}\right\|^{2} \nabla_{1}\left[\sum_{j=2}^{\infty} e^{B(1+\gamma j)}(j-1)\left\|P_{j}\right\|\right.}{\|z\|^{2}} \\
& \left.-\frac{(1-\gamma)(B-A)}{2(1+|B|)}\right] \leq 0,
\end{aligned}
$$

where

$$
\sum_{j=2}^{\infty} e^{B(1+\gamma j)}(j-1)\left\|P_{j}\right\| \leq \frac{(1-\gamma)(B-A)}{2(1+|B|)} .
$$

(3) For the case that $A=0$ and $B<0$, similar to (2), we get

$\left(\nabla_{1}+\nabla_{2}\right)(|I|-1)<\frac{1-B^{2}}{B-A} \frac{1+\cos \beta}{\cos \beta}$ 


$$
\begin{aligned}
& \cdot \frac{\left\|z_{0}\right\|^{2} \nabla_{1}}{\|z\|^{2}}\left[\sum_{j=2}^{\infty} e^{B\left|z_{1}\right|(1+\gamma j)}\left(1-\left|z_{1}\right|^{2}\right)^{j / 2-1}(j-1)\right. \\
& \left.\cdot\left\|P_{j}\right\|-\frac{(1-\gamma)(B-A)}{2(1+|B|)}\right] \leq \frac{1-B^{2}}{B-A} \frac{1+\cos \beta}{\cos \beta} \\
& \cdot \frac{\left\|z_{0}\right\|^{2} \nabla_{1}}{\|z\|^{2}}\left[\sum_{j=2}^{\infty}(j-1)\left\|P_{j}\right\|-\frac{(1-\gamma)(B-A)}{2(1+|B|)}\right] \\
& \leq 0,
\end{aligned}
$$

where

$$
\sum_{j=2}^{\infty}(j-1)\left\|P_{j}\right\| \leq \frac{(1-\gamma)(B-A)}{2(1+|B|)} .
$$

From (1) to (3) we get the desired conclusion.

Remark 18. Setting $A=-1=-B-2 \alpha$ and $A=-B=-\alpha$ in Theorems 15 and 17 and Corollary 16, respectively, we get the corresponding results for spiral-like mappings of type $\beta$ and order $\alpha$ and strong spiral-like mappings of type $\beta$ and order $\alpha$.

\section{The Invariance of Almost Spiral-Like Mappings of Type $\beta$ and Order $\alpha$}

In the following, we mainly discuss the perturbed Extension Operator (4) preserving the geometric characteristics of almost spiral-like mappings of type $\beta$ and order $\alpha$ on $\Omega$, and thus we get the conclusion on $B^{n}$ as well as the results about some subclasses.

Theorem 19. Let $f\left(z_{1}\right)$ be an almost spiral-like function of type $\beta$ and order $\alpha$ on $D$ with $\alpha \in[0,1 / 2) \cup(1 / 2,1)$ and $\beta \in(-\pi / 2, \pi / 2)$. Let $F(w, z)$ be the mapping denoted by (4) with $p_{i}>1, \delta_{i} \in[0,1](i=1, \ldots, s), \gamma \geq 0$ and $\left.\left(f\left(z_{1}\right) / z_{1}\right)^{\delta_{i}}\right|_{z_{1}=0}=1,\left.\left(f\left(z_{1}\right) / z_{1}\right)^{\gamma}\right|_{z_{1}=0}=1$. Let $P_{j}=0(j=$ $2,3)$ and $q \geq \delta /(n+1)$ where $\delta=\max \left\{p_{1} \delta_{1}, \ldots, p_{s} \delta_{s}\right\}$. Then $F(w, z)$ is an almost spiral-like mapping of type $\beta$ and order $\alpha$ on $\Omega$ provided that

$$
\begin{gathered}
\sum_{j=4}^{\infty}(j-1)\left\|P_{j}\right\|(2-2 \alpha)^{2(\alpha-1) /(2 \alpha-1)}(2 \alpha)^{(2(1-\alpha) /(2 \alpha-1)) \gamma j} \\
\cdot 2^{j / 2-1} \leq \frac{(1-\alpha)(1-\gamma) \cos \beta}{2(1-\alpha) \cos \beta+1}
\end{gathered}
$$

Proof. By Definition 3, we need to prove

$$
\begin{aligned}
& \Re\left[\frac{2}{\rho(w, z)} \frac{\partial \rho(w, z)}{\partial(w, z)} e^{-i \beta}(D F(w, z))^{-1} F(w, z)\right. \\
& \quad-\alpha \cos \beta] \geq 0,
\end{aligned}
$$

which is equivalent to

$$
\begin{aligned}
& \mathfrak{R}\left[(1-i \tan \beta) \frac{2}{\rho(w, z)} \frac{\partial \rho(w, z)}{\partial(w, z)}(D F(w, z))^{-1}\right. \\
& \quad \cdot F(w, z)-\alpha] \geq 0 .
\end{aligned}
$$

Similar to Theorem 11, the left side of (66) is the real part of a holomorphic mapping and thus is a harmonic function. Due to the minimum principle of harmonic functions, we need only to prove that (66) holds for $z \in \partial \Omega$ which implies that $\rho(w, z)=1$.

Let

$$
(1-i \tan \beta) \frac{f}{z_{1} f^{\prime}}-\alpha=h\left(z_{1}\right) .
$$

Since $f\left(z_{1}\right)$ is an almost spiral-like function of type $\beta$ and order $\alpha$ on $D$, then $h\left(z_{1}\right) \in H(D), \mathfrak{R} h\left(z_{1}\right)>0, h(0)=$ $1-i \tan \beta-\alpha$. Therefore $\mid\left(h\left(z_{1}\right)-(1-\alpha-i \tan \beta)\right) /\left(h\left(z_{1}\right)+\right.$ $(1-\alpha+i \tan \beta)) \mid<1$. Let

$$
g\left(z_{1}\right)=\frac{h\left(z_{1}\right)-(1-\alpha-i \tan \beta)}{h\left(z_{1}\right)+(1-\alpha+i \tan \beta)} .
$$

Then $\left|g\left(z_{1}\right)\right|<1, g(0)=0$. Moreover $\mathfrak{R} h\left(z_{1}\right)>0$ implies $h\left(z_{1}\right) \neq-(1-\alpha+i \tan \beta)$; thus $g\left(z_{1}\right) \in H(D)$. Applying Schwarz Lemma we obtain $\left|g\left(z_{1}\right)\right| \leq\left|z_{1}\right|$; in other words,

$$
\left|1+\frac{2(1-\alpha)}{h\left(z_{1}\right)-(1-\alpha-i \tan \beta)}\right| \geq \frac{1}{\left|z_{1}\right|},
$$

which leads to

$$
\left|h\left(z_{1}\right)-(1-\alpha-i \tan \beta)\right| \leq \frac{2(1-\alpha)\left|z_{1}\right|}{1-\left|z_{1}\right|} .
$$

Let

$$
\begin{aligned}
& (1-i \tan \beta) \frac{2 \partial \rho(w, z)}{\partial(w, z)}(D F(w, z))^{-1} F(w, z)-\alpha \\
& \quad=I .
\end{aligned}
$$

We then get the following equation string by (31) and (67):

$$
\begin{gathered}
\left(\nabla_{1}+\nabla_{2}\right)\left[(1-i \tan \beta) \frac{2 \partial \rho(w, z)}{\partial(w, z)}(D F(w, z))^{-1} F(w,\right. \\
z)-\alpha]=(1-i \tan \beta)\left\{\sum _ { k = 1 } ^ { s } p _ { k } \| w _ { ( k ) } \| ^ { 2 p _ { k } } \left[1-\delta_{k}\right.\right. \\
\left.. \frac{z_{1} f^{\prime}-f}{z_{1} f^{\prime}}\left(1+\frac{1}{f} \sum_{j=2}^{\infty}\left(\frac{f}{z_{1}}\right)^{\gamma j}(1-j) P_{j}\left(z_{0}\right)\right)\right]
\end{gathered}
$$




$$
\begin{aligned}
& +\frac{\left|z_{1}\right|^{2} \nabla_{1}}{\|z\|^{2}} \frac{f}{z_{1} f^{\prime}}\left[1+\frac{1}{f} \sum_{j=2}^{\infty}\left(\frac{f}{z_{1}}\right)^{\gamma j}(1-j) P_{j}\left(z_{0}\right)\right] \\
& +\frac{\left\|z_{0}\right\|^{2} \nabla_{1}}{\|z\|^{2}}\left[1-\gamma \frac{z_{1} f^{\prime}-f}{z_{1} f^{\prime}}(1\right. \\
& \left.\left.\left.+\frac{1}{f} \sum_{j=2}^{\infty}\left(\frac{f}{z_{1}}\right)^{\gamma j}(1-j) P_{j}\left(z_{0}\right)\right)\right]\right\}-\alpha\left(\nabla_{1}+\nabla_{2}\right) \\
& =(1-i \tan \beta) \nabla_{2}+\widetilde{\nabla_{2}}\left[h\left(z_{1}\right)-1+i \tan \beta+\alpha\right][1 \\
& \left.+\frac{1}{f} \sum_{j=2}^{\infty}\left(\frac{f}{z_{1}}\right)^{\gamma j}(1-j) P_{j}\left(z_{0}\right)\right]+\frac{\left|z_{1}\right|^{2} \nabla_{1}}{\|z\|^{2}}\left(h\left(z_{1}\right)\right. \\
& +\alpha)\left[1+\frac{1}{f} \sum_{j=2}^{\infty}\left(\frac{f}{z_{1}}\right)^{\gamma j}(1-j) P_{j}\left(z_{0}\right)\right]-\alpha\left(\nabla_{1}\right. \\
& \left.+\nabla_{2}\right)+\frac{\left\|z_{0}\right\|^{2} \nabla_{1}}{\|z\|^{2}}\left[1-i \tan \beta+\gamma\left(h\left(z_{1}\right)-1+i\right.\right. \\
& \left.\cdot \tan \beta+\alpha)\left(1+\frac{1}{f} \sum_{j=2}^{\infty}\left(\frac{f}{z_{1}}\right)^{\gamma j}(1-j) P_{j}\left(z_{0}\right)\right)\right] \\
& =(1-\alpha-i \tan \beta)(1-\gamma) \frac{\left\|z_{0}\right\|^{2} \nabla_{1}}{\|z\|^{2}}+(1-\alpha-i \\
& \cdot \tan \beta)\left(\nabla_{2}-\widetilde{\nabla_{2}}\right)+\left[\widetilde{\nabla_{2}}+\frac{\nabla_{1}}{\|z\|^{2}}\left(\left|z_{1}\right|^{2}+\gamma\left\|z_{0}\right\|^{2}\right)\right] \\
& \cdot h\left(z_{1}\right)+\left[\widetilde{\nabla_{2}}\left(h\left(z_{1}\right)-1+\alpha+i \tan \beta\right)\right. \\
& +\frac{\left|z_{1}\right|^{2} \nabla_{1}}{\|z\|^{2}}\left(h\left(z_{1}\right)+\alpha\right)+\frac{\gamma\left\|z_{0}\right\|^{2}}{\|z\|^{2}} \nabla_{1}\left(h\left(z_{1}\right)-1+\alpha\right. \\
& +i \tan \beta)] \frac{1}{f} \sum_{j=2}^{\infty}\left(\frac{f}{z_{1}}\right)^{\gamma j}(1-j) P_{j}\left(z_{0}\right)=(1-\alpha \\
& -i \tan \beta)(1-\gamma) \frac{\left\|z_{0}\right\|^{2} \nabla_{1}}{\|z\|^{2}}+(1-\alpha-i \tan \beta)\left(\nabla_{2}\right. \\
& \left.-\widetilde{\nabla_{2}}\right)+\left[\widetilde{\nabla_{2}}+\frac{\nabla_{1}}{\|z\|^{2}}\left(\left|z_{1}\right|^{2}+\gamma\left\|z_{0}\right\|^{2}\right)\right] h\left(z_{1}\right) \\
& +\left[\widetilde{\nabla_{2}}+\frac{\nabla_{1}}{\|z\|^{2}}\left(\left|z_{1}\right|^{2}+\gamma\left\|z_{0}\right\|^{2}\right)\right]\left(h\left(z_{1}\right)-1+\alpha+i\right. \\
& \cdot \tan \beta) \cdot \frac{1}{f} \sum_{j=2}^{\infty}\left(\frac{f}{z_{1}}\right)^{\gamma j}(1-j) P_{j}\left(z_{0}\right)+(1-i \tan \beta) \\
& \cdot \frac{\nabla_{1}\left|z_{1}\right|^{2}}{\|z\|^{2}} \frac{1}{f} \sum_{j=2}^{\infty}\left(\frac{f}{z_{1}}\right)^{\gamma j}(1-j) P_{j}\left(z_{0}\right) \text {. }
\end{aligned}
$$

Therefore, by (70), (38), and Lemma 10 there comes the following inequality string:

$$
\begin{gathered}
\left(\nabla_{1}+\nabla_{2}\right) \Re\left[(1-i \tan \beta) \frac{2 \partial \rho(w, z)}{\partial(w, z)}(D F(w, z))^{-1}\right. \\
\cdot F(w, z)-\alpha]=(1-\alpha)(1-\gamma) \frac{\left\|z_{0}\right\|^{2} \nabla_{1}}{\|z\|^{2}}+(1 \\
-\alpha)\left(\nabla_{2}-\widetilde{\nabla_{2}}\right)+\left[\widetilde{\nabla_{2}}+\frac{\nabla_{1}}{\|z\|^{2}}\left(\left|z_{1}\right|^{2}+\gamma\left\|z_{0}\right\|^{2}\right)\right]
\end{gathered}
$$$$
\cdot \mathfrak{R} h\left(z_{1}\right)+\mathfrak{R}\left\{\left[\widetilde{\nabla_{2}}+\frac{\nabla_{1}}{\|z\|^{2}}\left(\left|z_{1}\right|^{2}+\gamma\left\|z_{0}\right\|^{2}\right)\right]\right.
$$$$
\cdot\left(h\left(z_{1}\right)-1+\alpha+i \tan \beta\right) \frac{1}{f}
$$$$
\left.\cdot \sum_{j=2}^{\infty}\left(\frac{f}{z_{1}}\right)^{\gamma j}(1-j) P_{j}\left(z_{0}\right)\right\}+\Re\{(1-i \tan \beta)
$$$$
\left.\cdot \frac{\nabla_{1}\left|z_{1}\right|^{2}}{\|z\|^{2}} \frac{1}{f} \sum_{j=2}^{\infty}\left(\frac{f}{z_{1}}\right)^{\gamma j}(1-j) P_{j}\left(z_{0}\right)\right\} \geq(1-\alpha)
$$$$
\cdot(1-\gamma) \frac{\left\|z_{0}\right\|^{2} \nabla_{1}}{\|z\|^{2}}
$$$$
-\left\{\left[\widetilde{\nabla_{2}}+\frac{\nabla_{1}}{\|z\|^{2}}\left(\left|z_{1}\right|^{2}+\gamma\left\|z_{0}\right\|^{2}\right)\right] \frac{2(1-\alpha)}{1-\left|z_{1}\right|}\right.
$$$$
\left.+\frac{1}{\cos \beta} \frac{\nabla_{1}}{\|z\|^{2}}\right\} \cdot\left|\frac{z_{1}}{f}\right| \sum_{j=2}^{\infty}\left|\frac{f}{z_{1}}\right|^{\gamma j}(j-1)\left\|P_{j}\right\|\left\|z_{0}\right\|^{j}
$$$$
=(1-\alpha)(1-\gamma) \frac{\left\|z_{0}\right\|^{2} \nabla_{1}}{\|z\|^{2}}-\frac{\nabla_{1}}{\|z\|^{2}}
$$$$
\cdot \frac{1}{1-\left|z_{1}\right|}\left[\left(\frac{\widetilde{\nabla_{2}}}{\nabla_{1}}\|z\|^{2}+\left|z_{1}\right|^{2}+\gamma\left\|z_{0}\right\|^{2}\right) 2(1-\alpha)\right.
$$$$
\left.+\frac{1-\left|z_{1}\right|}{\cos \beta}\right] \cdot\left|\frac{z_{1}}{f}\right| \sum_{j=2}^{\infty}\left|\frac{f}{z_{1}}\right|^{\gamma j}(j-1)\left\|P_{j}\right\|\left\|z_{0}\right\|^{j} \geq(1
$$$$
-\alpha)(1-\gamma) \frac{\left\|z_{0}\right\|^{2} \nabla_{1}}{\|z\|^{2}}-\frac{\nabla_{1}}{\|z\|^{2}} \frac{1}{1-\left|z_{1}\right|}
$$$$
\frac{2(1-\alpha) \cos \beta+1}{\cos \beta}\left|\frac{z_{1}}{f}\right| \sum_{j=2}^{\infty}\left|\frac{f}{z_{1}}\right|^{\gamma j}(j-1)\left\|P_{j}\right\|\left\|z_{0}\right\|^{j}
$$$$
\geq(1-\alpha)(1-\gamma) \frac{\left\|z_{0}\right\|^{2} \nabla_{1}}{\|z\|^{2}}-\frac{\nabla_{1}}{\|z\|^{2}} \frac{1}{1-\left|z_{1}\right|}
$$$$
\cdot \frac{2(1-\alpha) \cos \beta+1}{\cos \beta}\left|\frac{z_{1}}{f}\right| \sum_{j=2}^{\infty}\left|\frac{f}{z_{1}}\right|^{\gamma j}(j-1)\left\|P_{j}\right\|\left\|z_{0}\right\|^{j}
$$

$\geq \frac{\left\|z_{0}\right\|^{2} \nabla_{1}}{\|z\|^{2}}\left\{(1-\alpha)(1-\gamma)-\frac{2(1-\alpha) \cos \beta+1}{\cos \beta}\right.$ 


$$
\begin{aligned}
& \sum_{j=2}^{\infty}(j-1)\left\|P_{j}\right\| \cdot\left[1+(1-2 \alpha)\left|z_{1}\right|\right]^{2(\alpha-1) /(2 \alpha-1)} \\
& \cdot\left[1-(1-2 \alpha)\left|z_{1}\right|\right]^{(2(1-\alpha) /(2 \alpha-1)) \gamma j}\left(1+\left|z_{1}\right|\right)^{j / 2-1} \\
& \left.\cdot\left(1-\left|z_{1}\right|\right)^{j / 2-2}\right\} \geq \frac{\left\|z_{0}\right\|^{2} \nabla_{1}}{\|z\|^{2}}\{(1-\alpha)(1-\gamma) \\
& -\frac{2(1-\alpha) \cos \beta+1}{\cos \beta} \sum_{j=2}^{\infty}(j-1)\left\|P_{j}\right\| \\
& \left.\cdot(2-2 \alpha)^{2(\alpha-1) /(2 \alpha-1)}(2 \alpha)^{(2(1-\alpha) /(2 \alpha-1)) \gamma j} 2^{j / 2-1}\right\},
\end{aligned}
$$

where $P_{j}=0(j=2,3)$ and

$$
\begin{gathered}
\sum_{j=4}^{\infty}(j-1)\left\|P_{j}\right\|(2-2 \alpha)^{2(\alpha-1) /(2 \alpha-1)}(2 \alpha)^{(2(1-\alpha) /(2 \alpha-1)) \gamma j} \\
\cdot 2^{j / 2-1} \leq \frac{(1-\alpha)(1-\gamma) \cos \beta}{2(1-\alpha) \cos \beta+1}
\end{gathered}
$$

This is equal to (66) and hence completes the proof.

Setting $w_{(1)}=\cdots=w_{(s)}=0$ in Theorem 19, we draw the following invariance of almost spiral-like mappings of type $\beta$ and order $\alpha$ under the generalized Roper-Suffridge operator (6) on the unit ball $B^{n}$ in $\mathbb{C}^{n}$.

Corollary 20. Let $f\left(z_{1}\right)$ be an almost spiral-like function of type $\beta$ and order $\alpha$ on $D$ with $\alpha \in[0,1 / 2) \cup(1 / 2,1)$ and $\beta \epsilon$ $(-\pi / 2, \pi / 2)$. Let $F(z)$ be the mapping denoted by (6) with $\gamma \geq 0$ and $\left.\left(f\left(z_{1}\right) / z_{1}\right)^{\gamma}\right|_{z_{1}=0}=1$. Let $P_{j}=0$ for $j=2,3$. Then $F(z)$ is an almost spiral-like mapping of type $\beta$ and order $\alpha$ on $B^{n}$ provided that

$$
\begin{gathered}
\sum_{j=4}^{\infty}(j-1)\left\|P_{j}\right\|(2-2 \alpha)^{2(\alpha-1) /(2 \alpha-1)}(2 \alpha)^{(2(1-\alpha) /(2 \alpha-1)) \gamma j} \\
\cdot 2^{j / 2-1} \leq \frac{(1-\alpha)(1-\gamma) \cos \beta}{2(1-\alpha) \cos \beta+1}
\end{gathered}
$$

Remark 21. Setting $\alpha=0$ and $\beta=0$ in Theorem 19 and Corollary 20, respectively, we get the corresponding results for spiral-like mappings of type $\beta$ and almost star-like mappings of order $\alpha$.

\section{Conflicts of Interest}

The authors declare that they have no conflicts of interest.

\section{Acknowledgments}

This work was supported by NSF of China (no. 11271359) and Science and Technology Research Projects of Henan Provincial Education Department (no. 17A110041).

\section{References}

[1] P. L. Duren, Univalent Functions, Springer, NewYork, NY, USA, 1983.

[2] H. Cartan, Sur la possibilité d'étendre aux fonctions de plusieurs variables complexes la theorie des fonctions univalents. Lecons sur les Fonctions Univalents ou Mutivalents, by P Montel, GauthierVillars, Paris, France, 1933.

[3] K. A. Roper and T. J. Suffridge, "Convex mappings on the unit

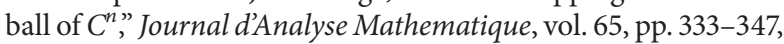
1995.

[4] I. Graham, G. Kohr, and M. Kohr, "Loewner chains and the Roper-Suffridge extension operator," Journal of Mathematical Analysis and Applications, vol. 247, no. 2, pp. 448-465, 2000.

[5] I. Graham and G. Kohr, "Univalent mappings associated with the Roper-Suffridge extension operator," Journal d'Analyse Mathematique, vol. 81, pp. 331-342, 2000.

[6] I. Graham, H. Hamada, G. Kohr, and T. J. Suffridge, "Extension operators for locally univalent mappings," Michigan Mathematical Journal, vol. 50, no. 1, pp. 37-55, 2002.

[7] S. Gong and T. Liu, "The generalized Roper-Suffridge extension operator," Journal of Mathematical Analysis and Applications, vol. 284, no. 2, pp. 425-434, 2003.

[8] I. Graham and G. Kohr, "The Roper-Suffridge extension operator and classes of biholomorphic mappings," Science in China. Series A. Mathematics, vol. 49, no. 11, pp. 1539-1552, 2006.

[9] J. Muir and T. J. Suffridge, "Unbounded convex mappings of the ball in $\mathbb{C}^{n}$," Proceedings of the American Mathematical Society, vol. 129, no. 11, pp. 3389-3393, 2001.

[10] J. Muir, "A modification of the Roper-Suffridge extension operator," Computational Methods and Function Theory, vol. 5, no. 1, pp. 237-251, 2005.

[11] G. Kohr, "Loewner chains and a modification of the RoperSuffridge extension operator," Mathematica, vol. 71, no. 1, pp. 41-48, 2006.

[12] J. Muir, "A class of Loewner chain preserving extension operators," Journal of Mathematical Analysis and Applications, vol. 337, no. 2, pp. 862-879, 2008.

[13] M. Elin and M. Levenshtein, "Covering results and perturbed Roper-Suffridge operators," Complex Analysis and Operator Theory, vol. 8, no. 1, pp. 25-36, 2014.

[14] X. Zhang, S. Feng, and Y. Li, "Loewner chain associated with the modified Roper-Suffridge extension operator," Computational Methods and Function Theory, vol. 16, no. 2, pp. 265-281, 2016.

[15] Y. Cui, C. Wang, and H. Liu, "The invariance of strong and almost spirallike mappings of type $\beta$ and order $\alpha$," Acta Mathematica Scientia. Series B, vol. 35B, no. 6, pp. 1454-1466, 2015.

[16] M. Elin, "Extension operators via semigroups," Journal of Mathematical Analysis and Applications, vol. 377, no. 1, pp. 239250, 2011.

[17] Y. Y. Tang, Roper-Suffridge operators on Bergman-Hartogs domain [Master, thesis], Henan University Master Thesis, Kaifeng, China, 2016.

[18] R. H. Cai and X. S. Liu, "The third and fourth coefficient estimations for the subclasses of strongly spirallike functions," Journal of Zhanjiang Normal College, vol. 31, pp. 38-43, 2010.

[19] C. L. Gao, The generalized Roper-Suffidge extension operator on a Reinhardt domain [Master, thesis], Zhejiang Normal University Master Thesis, Jinhua, China, 2012. 
[20] Y.-C. Zhu and M.-S. Liu, "The generalized Roper-Suffridge extension operator on REInhardt domain $D_{p}$," Taiwanese Journal of Mathematics, vol. 14, no. 2, pp. 359-372, 2010.

[21] T. Liu and G. Ren, "Growth theorem of convex mappings on bounded convex circular domains," Science in China. Series A. Mathematics, vol. 41, no. 2, pp. 123-130, 1998.

[22] H. Hamada and G. Kohr, "The growth theorem and quasiconformal extension of strongly spirallike mappings of type $\alpha$," Complex Variables, vol. 44, no. 4, pp. 281-297, 2001.

[23] J. F. Wang, "A growth theorem and the Roper-Suffridge extension operator for a class of starlike mappings in $C^{n}$," Chinese Annals of Mathematics, vol. 34A, no. 2, pp. 223-234, 2013.

[24] X.-s. Liu and S.-x. Feng, "A remark on the generalized RoperSuffridge extension operator for spirallike mappings of type $\beta$ and order $\alpha$," Chinese Quarterly Journal of Mathematics, vol. 24, no. 2, pp. 310-316, 2009. 


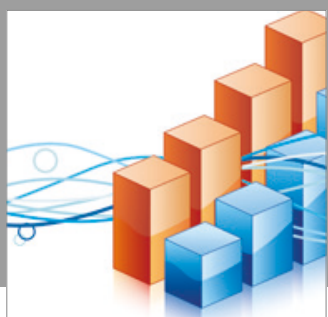

Advances in

Operations Research

vatersals

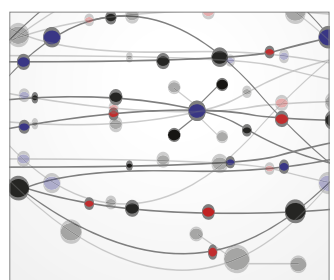

\section{The Scientific} World Journal
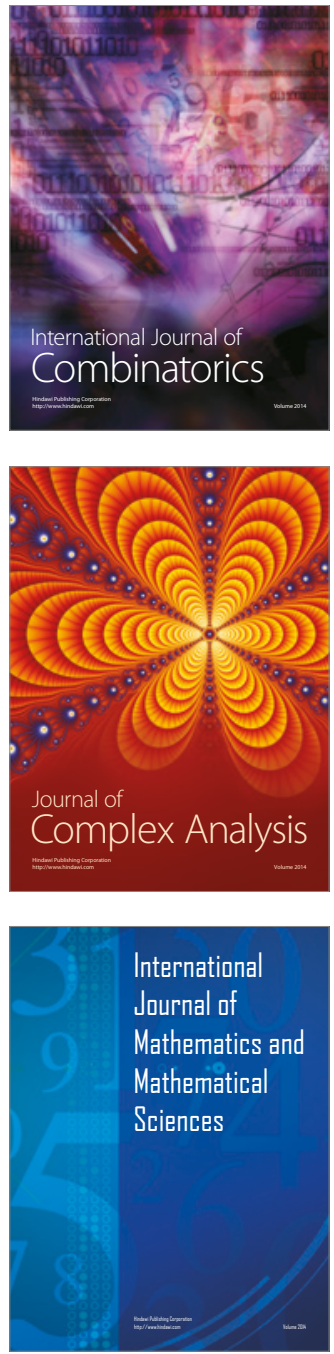
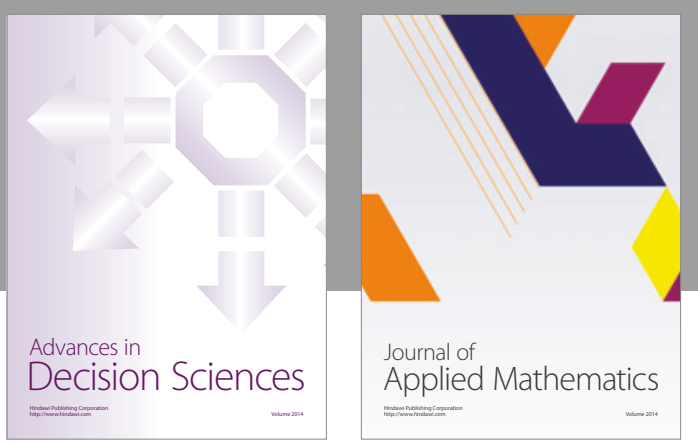

Algebra

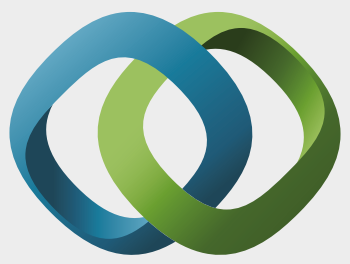

\section{Hindawi}

Submit your manuscripts at

https://www.hindawi.com
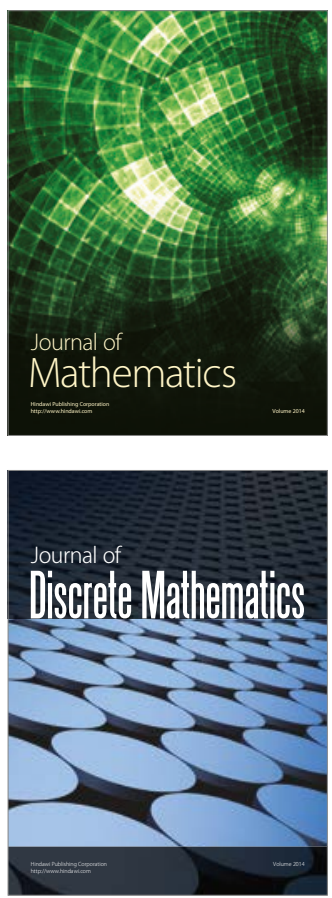

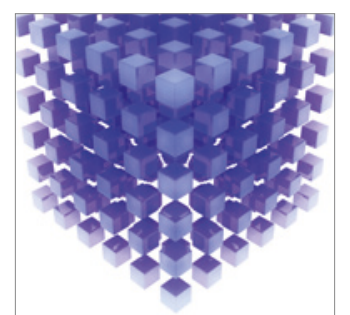

Mathematical Problems in Engineering
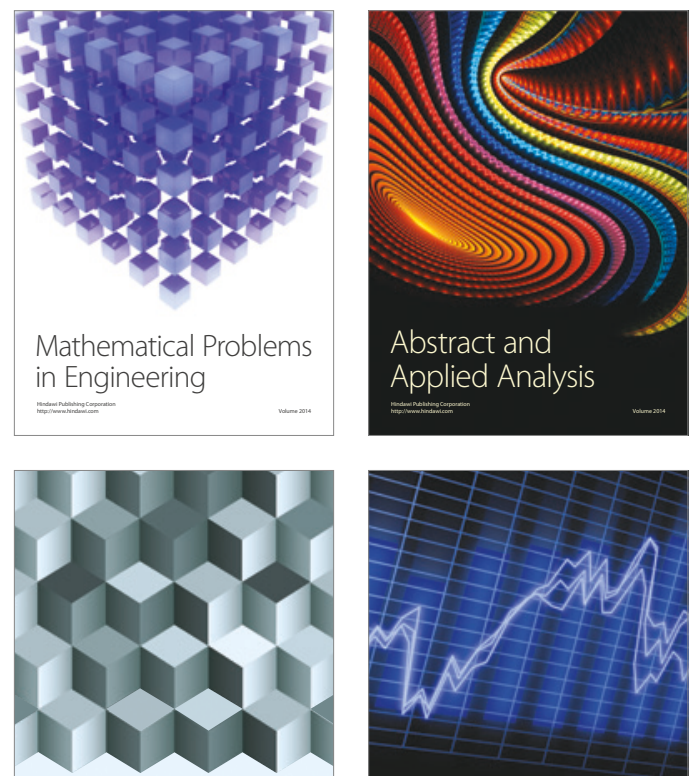

Journal of

Function Spaces

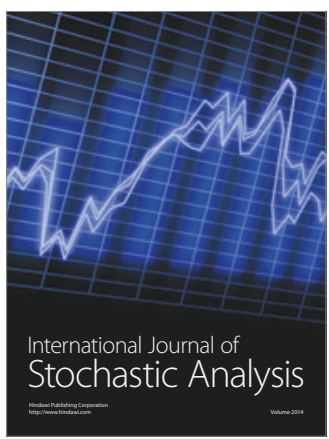

Probability and Statistics
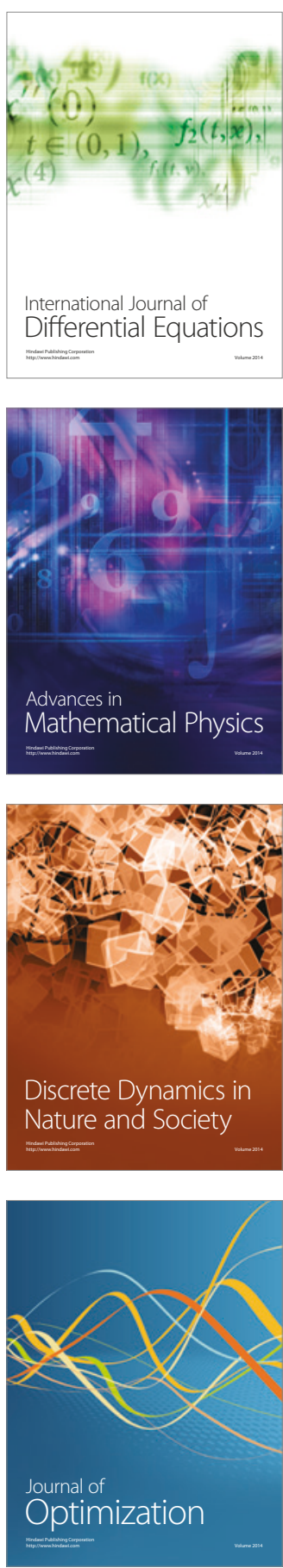